\title{
STATISTICAL THEORY OF PROPHYLACTIC AND THERAPEUTIC TRIALS \\ II. METHODS OF OPERATIONAL ADVANTAGE \\ BY
}

LANCELOT HOGBEN and RAYMOND WRIGHTON

\author{
Department of Medical Statistics, University of Birmingham, and Department of Social and Industrial Medicine, \\ University of Sheffield
}

\section{NeEd for a NeW Approach}

Statistical procedures may subserve either of two ends. In the conduct of government, commerce, and manufacture it may be legitimate to invoke them with no aim other than to prescribe a course of action which limits certain assignable risks. We speak appropriately of any such prescription as conditional. In biological research our primary concern is to establish propositions worthy to take their place in the corpus of scientific knowledge accepted as a basis for subsequent action unrestricted by immediate administrative preoccupations. We speak of any such assertion as unconditional. Much needless confusion concerning the credentials of statistical techniques arises through failure to recognize how far each is meaningful in one or other domain. Since our concern in this context is with the validification of results obtained in the conduct of scientific research, unconditional statistical inference alone is relevant to the end in view.

In our previous communication (Hogben and Wrighton, 1952), we have recognized a broad distinction between statistical procedures of two sorts, respectively referred to as tests and estimation. Under the first heading we have seen that it is now necessary to distinguish two prescriptions:

(i) the significance test, which operates within the framework of a unique null hypothesis;

(ii) the decision test, which involves the specification of alternative admissible hypotheses.

It is likewise necessary to distinguish between two ways in which contemporary writers use the term estimation, viz., point estimation and interval estimation. In either case, our concern is with a parameter (or parameters) of a particular universe from which we may draw a sample. Point estimation undertakes to specify a unique value of the parameter as the best one; but in doing so relinquishes the possibility of assigning an acceptable uncertainty safeguard to the form the assertion takes. Interval estimation repudiates the undertaking to specify any single value of it as better than every other. Within the framework of an acceptable level of uncertainty, i.e., probability of false assertion, it subsumes rules of procedure which entitle us to make statements delimiting a range of values within which the parameter lies.

In the opening paragraph of this contribution, and elsewhere in the previous one, we have drawn an admittedly provisional distinction between conditional and unconditional assertions in terms of the uses to which we put them. This is clear-cut in the sense that:

(a) any statement worthy to rank in the corpus of scientific knowledge is one which we can rightly describe as unconditional in the sense elsewhere defined;

(b) statements of the conditional sort may suffice as a basis for administrative decision.

It is none the less possible to formulate rules of decision leading to unconditional statements of a sort rarely, if ever, relevant to the domain of research in pure science and no more useful to the administrator because more comprehensive in scope than a corresponding statement expressed in the more restricted form. Such is the class of decision tests which emerge in the theory of consumer and producer risk.

Further consideration of the Drosophila model of our earlier contribution will make this clear. We there set up two hypotheses: $H_{a}$ that $p=\frac{1}{2}=p_{a}$, and $H_{b}$ that $p=\frac{2}{3}=p_{b}, p$ being the probability that any offspring of a particular mother will be female. If we make the rule to reject $H_{a}$ if $x>\left(a+\frac{1}{2}\right)$ for the $r$-fold sample, denoting by $L_{x \cdot a}$ the probability that it will contain $x$ females if $p=p_{a}$, we may assign as the conditional risk $(\alpha)$ of rejection when $H_{a}$ is true:

$$
\alpha=\sum_{x=(a+1}^{x=r} L_{x \cdot a}
$$


Similarly, we may adopt $H_{b}$ as our null hypothesis and make the rule to reject it if $x<\left(b+\frac{1}{2}\right)$. The corresponding conditional risk $(\beta)$ of rejection is then:

$$
\beta=\sum_{x=0}^{x=b} L_{x \cdot b} .
$$

In either case, we attach an uncertainty safeguard $(\alpha$ or $\beta)$ to a statement which is conditional in the sense that it refers to a risk we take of being wrong if a particular hypothesis is correct. Unless $a=b$, the simultaneous application of the two rejection criteria will not necessarily lead to a decision in favour of either hypothesis; but we can formulate a composite rule which must do so in the form:

$$
\begin{aligned}
& \text { reject } H_{a} \text { if } x>\left(k+\frac{1}{2}\right) \\
& \text { reject } H_{b} \text { if } x<\left(k+\frac{1}{2}\right) .
\end{aligned}
$$

We may then be able to choose $k$ so that $\alpha \simeq \gamma \simeq \beta$, if $r$ is fairly large. This leads to a conditional assertion which assigns $\gamma$ as the risk that we shall reject either hypothesis if true; but it does not assign an acceptable safeguard to any unconditional assertion about the outcome unless $H_{a}$ and $H_{b}$ are the only admissible hypotheses. We can make a more comprehensive type of statement if we restate our hypothesis in the form $H_{a}$ that $p \leqslant p_{a}$ and $H_{b}$ that $p \geqslant p_{b}$; and may still guarantee the termination of the test in a decision if we follow the same composite rule of rejecting $H_{a}$ when $x>\left(k+\frac{1}{2}\right)$ and rejecting $H_{b}$ when $x<\left(k+\frac{1}{2}\right)$. We may then define $L_{x \cdot a}$ and $L_{x \cdot b}$ in terms of $p_{a}$ and $p_{b}$ as before, and fix $k$ so that:

$$
\sum_{x=(k+1)}^{x=r} L_{x \cdot a} \simeq \alpha \simeq \sum_{x=0}^{x=k} L_{x \cdot b} .
$$

Any value $p<p_{a}$ then makes the conditional risk of rejecting $H_{a}$ in its new form less than $\alpha$; and any value of $p>p_{b}$ makes the conditional risk of rejecting $H_{b}$ in its new form less than $\alpha$, which we may assign at any acceptable level, if free to prescribe in advance the sample size. The rule itself limits our allowable positive statements to the alternatives $p>p_{a}$ and $p<p_{b}$. Except for the trivial case $p_{a}=0$ or $p_{b}=1$, it prohibits any statement of the form $p_{a} \leqslant p \leqslant p_{b}$. Since $\alpha$ sets a limit to the probability of any false assertion we may make, we are entitled to say that $P_{f} \leqslant \alpha$ unconditionally defines the uncertainty safeguard of the entire class of statements which the rule subsumes; but we can state this only because the rule subsumes no possibility of simultaneous statement concerning the relation of $p$ to both $p_{a}$ and $p_{b}$.

If we know that the Drosophila culture contains several different genotypes to which we can assign values of $p$, we can meaningfully postulate prior probabilities referable to existent populations at risk to formalize the unconditional character of the final statement which the rule endorses. We must do so with due regard to its content, viz.: the probability of wrongly rejecting the hypothesis $p_{a}<p<p_{b}$ is zero, since the rule does not allow us to reject it. We may then set out the argument in terms of the following symbols, $\varepsilon$ being positive:

\begin{tabular}{c|c|l}
\hline Hypothesis & Prior Probability & $\begin{array}{c}\text { Conditional } \\
\text { Uncertainty Safeguard }\end{array}$ \\
\hline (1) $p<p_{a}$ & $P_{1}$ & $P_{f}=\alpha-\varepsilon_{1}$ \\
(2) $p=p_{a}$ & $P_{2}$ & $P_{f}=\alpha$ \\
(3) $p_{a}<p<p b$ & $P_{3}$ & $P_{f}=0$ \\
(4) $p=p_{b}$ & $P_{4}$ & $P_{f}=\alpha$ \\
(5) $p>p_{b}$ & $P_{5}$ & $P_{f}=\alpha-\varepsilon_{5}$ \\
& &
\end{tabular}

These hypotheses constitute an exclusive set of which our verdict can embody the acceptance of only one. Hence the addition rule applies, and our unconditional uncertainty safeguard is:

$$
\begin{aligned}
P_{f}= & P_{1} \cdot P_{f \cdot 1}+P_{2} \cdot P_{f \cdot 2}+P_{3} \cdot P_{f .3}+P_{4} \cdot P_{f .4}+P_{5} \cdot P_{f .5} \\
= & P_{1}\left(\alpha-\varepsilon_{1}\right)+P_{2} \cdot \alpha+P_{4} \cdot \alpha+P_{5} \cdot\left(\alpha-\varepsilon_{5}\right) \\
= & \left(1-P_{3}\right) \alpha-P_{1} \cdot \varepsilon_{1}-P_{5} \cdot \varepsilon_{5} \\
& \therefore P_{f} \leqslant \alpha .
\end{aligned}
$$

The prescription of such a rule presupposes two target values of $p$. These we can readily conceive in relation to standards of quality and to costing limits in an executive set-up, but the unconditional form the terminal statement assumes when we formulate a rule in this way embodies no relevant information other than the content of two types of conditional assertion. What the choice of a single acceptance-rejection criterion-score $k$ accomplishes is that a statistical inspection plan then achieves its task, i.e., the test must lead to a decision to reject either $H_{a}$ or $H_{b}$. In fact, both hypotheses may be wrong; and the unconditional form of the assertion is realizable only because the test can never lead to a corresponding assertion, i.e., a statement of the form $p_{a}<p<p_{b}$.

If we operate within the framework of a single hypothesis stated in the form $p \leqslant p_{a}$ or $p \geqslant p_{b}$, and have defined our rejection criterion so that $P_{f} \leqslant \alpha$ is the probability of rejecting it when true, we are free to limit our verdicts, as Fisher (1949)* does indeed prescribe, to the alternatives: hypothesis false and hypothesis unproven. In the sense that $P_{f} \leqslant \alpha$ is then the probability of erroneously making an allowably decisive assertion, we might admittedly say that $P_{f} \leqslant \alpha$ is the unconditional safeguard of our test procedure. We then evade the Neyman-Pearson error of the second kind by exposing ourselves to situations in which the overwhelming

* "It should be noted that the null hypothesis is never proved or established but is possibly disproved in the course of experimentation." ("The Design of Experiments", 5th ed., 1949, p. 16.) 
majority of our decisions will assign the verdict unproven to a false null hypothesis. We can indeed avoid doing so only by prescribing sample size with due regard to the Neyman-Pearson concept of test power; but any attempt to rehabilitate the Yule-Fisher significance test on such terms undermines previous claims concerning the value of inference referable to small samples.

That the distinction between conditional and unconditional is not so clear-cut, as we have provisionally assumed in the foregoing contribution, therefore emphasizes the importance of examining the advantages or drawbacks of any statistical procedure with due regard to the type of terminal statements which it can or cannot endorse. One puzzling feature of a test procedure which operates within the framework of a unique null hypothesis arises from the naïve assumption that the appropriate form of the latter is in the words of Fisher (1949), the "hypothesis that the phenomenon to be demonstrated is in fact absent". Current practice interprets this to signify that the true difference with reference to treatment efficacy is zero. All the test can thus achieve is to assess the risk of accepting one treatment as better than another when they are equally good. Unless it is clear that there exists on this planet a body of persons actively interested in a rule of procedure with such terms of reference, it can lead only to statements which are either noncommittal or irrelevant.

The administrator concerned with allocation of costly resources will wish to know whether Treatment $B$ is at least so much better than Treatment $A$. The physician anxious to invoke any means of possible benefit will ask the same sort of question but set his target value at a lower level. The manufacturer eager to exploit a new prospect but alert to the danger of losing goodwill may wish to balance risks of two sorts by a dual test procedure such as the foregoing; and the research worker who invokes a statistical device to validate his findings will do so because he rightly or wrongly believes in its relevance to some form of unconditional assertion about how much the efficacy of Treatment $B$ exceeds that of Treatment $A$.

Commonly, we shall not undertake a trial unless prior sources of information such as experiments in vitro or on animals have given us good reason to suppose that the new Treatment $B$ is more efficacious than Treatment $A$. On that understanding, our practical interest in the outcome may be:

(a) to ensure that the patient has the benefit of a new Treatment $B$ if its efficacy may exceed that of Treatment $A$ by as much as $x_{1}$; (b) to avoid substituting Treatment $B$ for Treatment $A$ at a cost disproportionate to the benefit conferred unless the efficacy of Treatment $B$ exceeds that of Treatment $A$ by at least $x_{2}$.

We subsume both objectives in the type of statement with which interval estimation deals, namely $x_{2} \leqslant d \leqslant x_{1}$; but we then relinquish the right to fix $x_{2}$ and $x_{1}$ in advance. Alternatively, we may adopt the dual test approach, e.g. we may equalize the risks $(\alpha=\beta)$ of rejecting Treatment $B$ as a better substitute if at least $x_{1}$ is 10 per cent. more effective than Treatment $A$ and of accepting it as a better substitute if no more than $x_{2}$ is 2 per cent. better than Treatment $A$ in the same sense. Inter alia, we may then ask:

Is a sequential procedure based on such a choice preferable to the method of interval estimation?

The question so stated is of topical interest, because it seemingly discloses the prospect of more rapid appraisal of treatment efficacy; but this hope may be illusory. We have first to suppose that the immediate assessment of efficacy is practicable pari passu with the assembly of data. We have also to suppose that the investigator can prescribe acceptable numerical values of $x_{1}$ and $x_{2}$. Aside from this, there is an as yet unresolved difficulty to face. The particular method for comparing two binomial parameters $\left(p_{b}\right.$ and $\left.p_{a}\right)$ put forward by Wald (1947), takes as its criterion of relative efficacy the ratio $u=p_{b}\left(1-p_{a}\right) \div p_{a}\left(1-p_{b}\right)$. The pivotal hypotheses are then definable in terms of agreed values $\left(u_{0}\right.$ and $\left.u_{1}\right)$ this ratio may attain. In the therapeutic trial, however, the relevant criterion (vide p. 219 infra) is the difference $d=p_{b}-p_{a}$, and we cannot express $u_{0}$ and $u_{1}$ in terms of agreed alternative values $d_{0}$ and $d_{1}$ unless we know the true value of $p_{a}$ (or $p_{b}$ ) in advance.

If we can indeed prescribe target values $x_{1}$ and $x_{2}$ in numerical terms, we are free to state the problem in the framework of alternative risks and to design the trial with due regard to economy of material; but those responsible for the design of a trial are rarely, if ever, in the position to do so. The investigator who claims to pursue truth for its own sake will prefer a procedure leading to some form of unconditional assertion concerning a range of values within which $d$ lies. To design a useful trial economically, he must then be able to specify how short the interval must be. This presupposes background knowledge outside the scope of statistical theory. Otherwise he can merely hope to make the interval $x_{2}-x_{1}$ as small as the expenditure of available materials permits and to locate it with due regard to the end in view. We may then 
explore the possibility of designing the trial in terms of treatment-group size to ensure that the length of the interval will not exceed an acceptable limit; but this presupposes some ulterior criterion of acceptable accuracy.

In our view, interval estimation is the one available procedure which offers the prospect of statistical validification of judgments which are the chief concern of the research worker in the conduct of the clinical trial. Its neglect in the domain of medical statistics would, therefore, be difficult to explain, if it were not also true that the basic postulates involve:

(i) a radical departure from the concept of point estimation which is traceable to the LegendreGauss theory of errors;

(ii) an overdue reorientation of our views concerning the nature of statistical validification.

Because the new approach associated with the term confidence interval is still novel in the context of the clinical trial, it will not be profitless if we set forth its implications between two schools of doctrine against the background of simple statistical models with numerical illustrations.

The development of the theory is largely due to J. Neyman; but credit for an early explicit statement of a procedure appropriate to large samples in the domain of taxonomic scoring is due to Wilson (1927). Since Wilson's contribution has received little recognition, it will not be out of place to quote his words from a later paper (Wilson, 1942):

In 1927 I called attention to the fact that many statements about probability are highly elliptical and illustrated the matter by the simple case of a pointbinomial universe with unknown probability $p$ and observed value $p_{o}$ in some sample. Using the admittedly rough estimate of probability based on the standard deviation one ordinarily writes:

$$
p_{o}-\lambda \sqrt{\overline{p_{o} q_{o} / n}}<p<p_{o}+\lambda \sqrt{p_{o} q_{o} / n} \text {. }
$$

and states that the probability that the true value $p$ in the universe lies between the limits given may be had from a probability-integral table entered with a normal deviation of $\lambda$ units. I urged that a better procedure would be to use for the standard deviation the value $p q / n$ obtained from the unknown $p$ of the universe which leads to:

$$
\begin{aligned}
\frac{p_{o}+t / 2}{1+t}-\frac{\sqrt{p_{o} q_{o} t+t^{2} / 4}}{1+t}<p & <\frac{p_{o}+t / 2}{1+t} \\
& +\frac{\sqrt{p_{o} q_{o} t+t^{2} / 4}}{1+t} .
\end{aligned}
$$

\section{One Approach to Confidence Theory}

One may approach the method of interval estimation against the background of two types of model situation. The more direct accepts the factual restriction of the topic to a single though unknown universe of choice; and is therefore under no obligation to introduce the nebulous prior probabilities of Bayes's theorem which rightly pertains only to situations admitting sampling in two stages. Alternatively, we may conceptualize it in terms of a Bayes's situation to make their irrelevance more explicit. The model we shall then invoke will also help us both to materialize the relation of interval estimation to the new theory of test procedure and to exhibit this relation as one to which the concept of prior probability, once elevated to a more commanding status in the theory of statistical inference, is also irrelevant. Our first series of models illustrates the direct approach.

Model I $(a)$.-We shall conceive that a lottery wheel has 1,024 sectors labelled with scores $x,(x+1),(x+2)$, $(x+3) \ldots(x+9),(x+10)$, respectively allocated to $1,10,45,120,210,252,210,120,45,10,1$ sectors. So much we know; but we do not know the numerical value of $x$ itself. At each spin we record as our score that of the sector opposite a fixed pointer. We now suppose that we spin the wheel forty times and record the mean score $\left(M_{x}\right)$ of the 40 -fold sample. Our problem is to define what we can legitimately assert about $x$. We shall first assemble available information relevant to the formal solution.

The long-run mean value $(M)$ of the score of any sample is, of course, $(x+5)$; and the terms of $\left(\frac{1}{2}+\frac{1}{2}\right)^{10}$ define the unit sample distribution (u.s.d.) of the universe with variance $\sigma^{2}=2 \cdot 5$, whence that of the distribution of the 40 -fold sample mean is:

$$
\sigma_{m}^{2}=\frac{\sigma^{2}}{40}=\frac{1}{16} .
$$

Thus $\sigma_{m}=0.25$; and the error involved in a normal quadrature for the distribution of the sample means is trivial. We can thus say that:

(a) the mean $\left(M_{x}\right)$ of 2.5 per cent. of all samples in the long run will exceed $M+2 \sigma_{m}=M+0 \cdot 5$;

(b) the mean of 2.5 per cent. of all samples in the long run will be less than $M-2 \sigma_{m}=M-0.5$;

(c) the mean of 95 per cent. of all samples will lie in the range $M \pm 2 \sigma_{m}=M \pm 0 \cdot 5$.

We now prescribe the following rule. We shall consistently disregard any values of $M_{x}$ if they lie outside the range $M \pm 2 \sigma_{m}$, thus asserting of any sample within our experience that:

$$
M-2 \sigma_{m} \leqslant M_{x} \leqslant M+2 \sigma_{m} \quad \ldots \quad \ldots
$$

If we do follow this rule consistently, 95 per cent. of our assertions will be true in the long run, i.e., within the framework of an indefinitely protracted series of trials. Now the foregoing is equivalent to the alternative assertion:

$$
M_{x}+2 \sigma_{m} \geqslant M \geqslant M_{x}-2 \sigma_{m} . \ldots \quad \ldots
$$

Thus 95 per cent. of our assertions will also be correct if we say that $M$ lies within the range of values so defined. We can set out the above reasoning in tabular form (opposite). 


\begin{tabular}{|c|c|c|c|}
\hline Event & $\begin{array}{l}\text { Probability of } \\
\text { its Occurrence }\end{array}$ & Equivalent Assertion & $\begin{array}{l}\text { Probability of } \\
\text { its Truth }\end{array}$ \\
\hline $\begin{aligned} M_{x} & >M+2 \sigma_{m} \\
M_{x} & <M-2 \sigma_{m} \\
M-2 \sigma_{m} & \leqslant M_{x} \leqslant M+2 \sigma_{m}\end{aligned}$ & $\begin{array}{l}0.025 \\
0.025 \\
0.95\end{array}$ & $\begin{array}{c}M<M_{x}-2 \sigma_{m} \\
M>M_{x}+2 \sigma_{m} \\
M_{x}+2 \sigma_{m} \geqslant M \leqslant M_{x}-2 \sigma_{m}\end{array}$ & $\begin{array}{l}0.025 \\
0.025 \\
0.95\end{array}$ \\
\hline
\end{tabular}

Let us now suppose that a recorded result of a 40 -fold spin is that the mean score is $6 \cdot 3$, and that we have no other information at our disposal. If we proceed consistently within the framework of our rule, we shall say that we attach a 5 per cent. uncertainty safeguard to the assertion: $M$ lies within the range $6 \cdot 3 \pm 0.5$ or $5 \cdot 8$ to $6 \cdot 8$. Since $M=x+5$ by definition, we can say with equal confidence that $x$ itself lies within the range 0.8 to 1.8 inclusive, assigning 1 as the correct value (at the 95 per cent. confidence level), if $x$ is an integer.

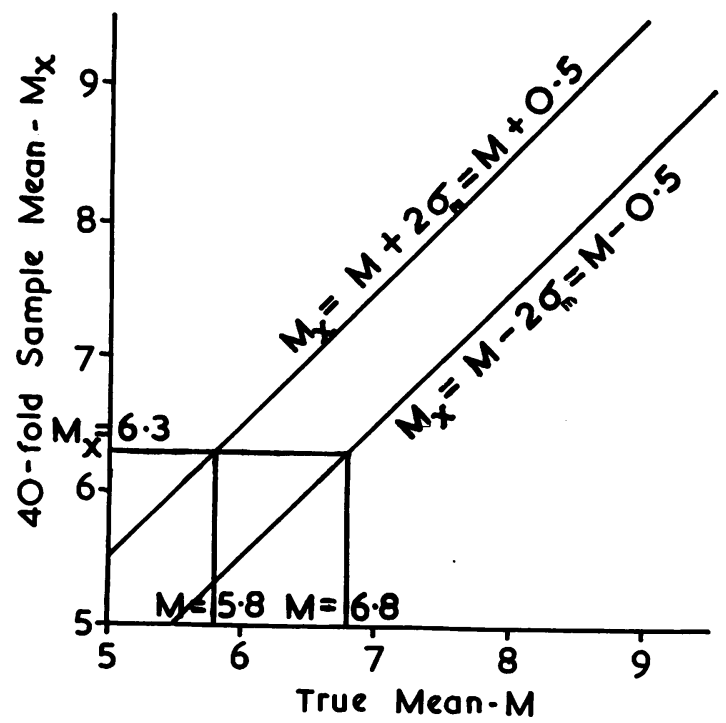

Fig. 1.-Graphical representation of the two-sided confidence limits for the sample mean of a normal variate of known variance (Model I.a).

Fig. 1 exhibits the argument based on our lottery wheel model within the range of values $5 \leqslant M \leqslant 9$ and $0 \leqslant x \leqslant 4$. For any value of $M$ we deny the occurrence of all values of $M_{x}$ greater than $M+2 \sigma_{m}$ or less than $M-2 \sigma_{m}$ with a probability of erroneous rejection approximately equal to 0.05 . Thus 95 per cent. of all sample values of $M_{x}$ will lie within the two parallel lines $M_{x}=M+2 \sigma_{m}$ and $M_{x}=M-2 \sigma_{m}$. There will correspond to any observed value of $M_{x}\left(\right.$ e.g., $\left.M_{x}=6 \cdot 3\right)$ two values of $M_{x}\left(5 \cdot 8\right.$ and 6.8 if $\left.M_{x}=6 \cdot 3\right)$ where the line through $M_{x}$ parallel to the abscissa cuts these two lines. These two values will define the range of $M$ consistent with the probability of error assigned to our denial of the limits of admissible values of $M_{x}$. The specification of the probability of error, i.e., uncertainty safeguard, being in this context 5 per cent. presupposes that we follow the rule regardless of the structure of any particular sample. In one sense, therefore, we imply the existence of a rule stated in advance of the examination of the data. This pinpoints the reorientation referred to earlier. It is misleading to speak of statistical validification as a technique for weighing the evidence any single sample supplies. It would be more correct to say that statistical theory weighs the ways in which we propose to weigh evidence supplied by samples.

In one respect, the foregoing model is highly artificial, i.e., we know in advance the numerical value of the variance $(\sigma)$ of the $u . s . d$. and hence that of $\sigma_{m}$. When sampling from a putatively normal universe we rarely, if ever, have such knowledge; but we do know the distribution of the ratio $\left(M_{x}-M\right)$ to the unbiassed sample estimate $\left(s_{m}\right)$ of $\sigma_{m}$. The $t$-function specifies the sample distribution of the ratio of these two sample statistics. Hence we can get from the $t$-table upper and lower limits for $M$ consistent with any assigned probability of erroneous statement within the framework of repeated application of the rule; and we can do the same for $\sigma^{2}$ itself by recourse to tables of the $\chi^{2}$ distribution.

If (as usually) we do not know the exact value of $\sigma_{m}$ but only the estimate $s_{m}$ based on an $r$-fold sample, we can use the $t$-ratio with $(r-1)$ degrees of freedom:

$$
t=\frac{\left(M_{x}-M\right)}{S_{m}} \text {. }
$$

The column for $(r-1)$ degrees of freedom gives the value $\pm t_{0.05}$ of $t$ such that $P=0.05$ is the probability that $t$ lies outside these limits. We can thus say of 95 per cent. samples that:

$$
t=\frac{M_{x}-M}{S_{m}} \text { lies within the range } \pm t_{0 \cdot 05} .
$$

Of 95 per cent. samples we can thus say that:

$$
\left(M_{x}-s_{m} \cdot t_{0 \cdot 05}\right) \leqslant M \leqslant\left(M_{x}+s_{m} \cdot t_{0 \cdot 05}\right) \text {. }
$$

Whence with a 5 per cent. uncertainty safeguard we can assert that:

$$
M_{x}-s_{m} \cdot t_{0 \cdot 05} \leqslant M \leqslant M_{x}+s_{m} \cdot t_{0 \cdot 05} \quad \ldots
$$

Model I (b).-Our last model invokes the system of scoring distinguished as representative in the previous contribution of this series. Our concern is then commonly with the sample mean of a set of measurements or counts. Our next model illustrates the confidence approach to estimation in the domain of taxonomic scoring, as when we estimate the proportion of affected in a treatment group. We now suppose that our lottery wheel has 100 sectors on each of which the number of pips is either 0 or 1 . We do not know the number [100q] of sectors which carry no pips, or the number $[100 p=$ $100(1-q)]$ of sectors which carry one pip. We spin 
it one hundred times and record the mean score. Our problem is to define confidence limits of $p$, the proportion of sectors which carry one pip. We are here sampling in an infinite two-class universe, and successive terms of $(q+p)^{100}$ define the frequencies of the observed proportionate (mean) score $p_{o}=0,0.01,0.02,0.03 \ldots$ $0.09,1 \cdot 0$. The unknown variance of the distribution of $p_{o}$ is given by:

$$
\sigma_{p}^{2}=\frac{p(1-p)}{100}
$$

Throughout the range of prescribed values, from $p=0.1$ to $p=0.9$ inclusive, the distribution of the observed proportionate score will be approximately normal. The range $p_{o}=p \pm 2 \sigma_{p}$ will therefore define the 95 per cent. confidence level well enough for expository purposes. Since $\sigma_{p}$ depends on $p$ being zero when $p=0$ or $p=1$, the two boundaries of acceptable values of $p_{o}$ will not be parallel straight lines as in Fig. 1 . They will meet at $p=0$ and $p=1$, the upper being concave downwards, the lower being concave upwards, as in Fig. 2. The corresponding acceptable range of $p$ values for any observed value of $p_{o}$ is unobtainable graphically, as before, by drawing a horizontal line parallel to the abscissa; but each limit is subsumed by the two roots of the quadratic:

$$
\left(p_{o}-p\right)^{2}=4 \sigma_{p}^{2}=\frac{p q}{25} \text {. }
$$

If the observed mean value is 0.62 , this becomes:

$$
\begin{array}{r}
25(0.62-p)^{2}=p(1-p), \\
\therefore 104 p^{2}-5 \cdot 24 p+38 \cdot 44=0, \\
\therefore \quad p \simeq 0.52 \text { or } 0.71 .
\end{array}
$$

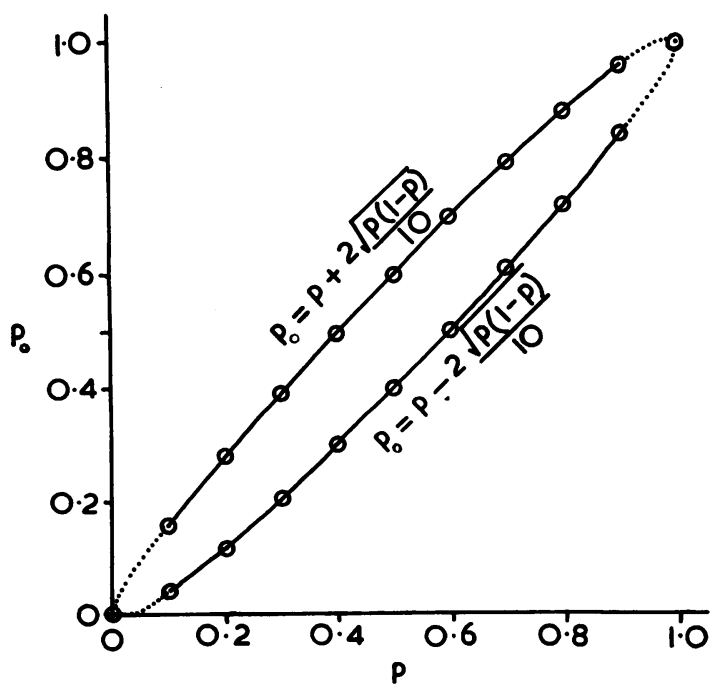

FIG. 2.-Graphical representation of the two-sided confidence limits for the proportionate score referable to a large sample from a two-class universe (Model I. $b$ ).

Note.-The dotted line in the terminal regions is to remind the reader that the normal approximation will hold good near the limits of the range only if the samples are very large.
At the $2 \sigma$ (95 per cent.) confidence level, we shall therefore say that our lottery wheel has no more than 71 and no less than 52 sectors carrying one pip. Alternatively, we attach 0.05 as our uncertainty safeguard to the assertion. More generally, we may set our limits for admissible values of $p_{o}$ in the range $p \pm h \sigma_{p}$, so that the appropriate quadratic for $r$-fold samples is:

$$
\left(p_{o}-p\right)^{2}=h^{2} \sigma_{p}^{2}=\frac{h^{2} p(1-p)}{r} .
$$

Whence we obtain Wilson's solution:

$$
p=\frac{\left(2 r p_{o}+h^{2}\right)}{2\left(r+h^{2}\right)} \pm \frac{h \sqrt{h^{2}+4 r p_{o}\left(1-p_{o}\right)}}{2\left(r+h^{2}\right)} \ldots
$$

If the size of the sample is small, we can define for any value of $p$ limits which exclude a proportion equal to or less than $2 \cdot 5$ per cent. (or other agreed figure) at either end of the range by recourse to the tables of the binomial (Clopper and Pearson, 1934; National Bureau of Standards, 1949). When we are comparing prophylactic or therapeutic measures with a low rate of attack or a high proportion of cures as the case may be, $p$ is by definition near to zero, or to unity, in which event the condition implicit in (ii) will not hold good, unless the size of the sample is very large. Even so, the order of error is difficult to assess when we invoke a continuous distribution such as the normal as a computing device for quadrature in the domain of discrete score values. This will come into focus in the next model situation we shall examine. We shall then see more clearly why we must confine statements about the confidence level to the form $P_{f}<\alpha$ or $P_{f} \leqslant \alpha$ when the variate is discrete, as is always true of the taxonomic method of scoring most commonly used in therapeutic or prophylactic trials.

MODEL I (c).--It limits our horizon unduly, if we confine our interpretation of confidence limits to situations in which we can assume without appreciable error that our score distribution is approximately normal and the confidence interval itself expressible in terms of its variance. The latter has no relevance when the universe is rectangular; and we may therefore deepen our insight into the logic of confidence theory, if we now lay aside any preoccupation with the normal distribution. As an elementary example of the confidence approach to estimation in the rectangular universe, we may consider the following model. A lottery wheel has $s$ sectors with consecutive scores from 1 to $s$, so that the proportion of sectors whose score value $(x)$ exceeds $m(\leqslant s)$ is $(s-m) \div s$. We shall suppose that we spin it once and record $x$. Our first problem will be: what can we legitimately say about $s$ ?

In the treatment of the foregoing model, we have side-stepped a limitation of interval estimation in the domain of discrete score values by assuming 
a good enough normal fit. Unless we postulate a continuous distribution we cannot in fact assign an uncertainty safeguard $\left(P_{f}=\alpha\right)$ or confidence level $\left(1-P_{f}\right)=(1-\alpha)$ to an admissible range of score values. The best we can assert is a statement of the form $P_{f} \leqslant \alpha$ or $P_{f}<\alpha$, as when we use tables of the binomial in the situation of ModeL $I(b)$. One reason for this is that we can assign more than one value to $m$ consistent with a fixed value $P_{f}=\alpha$ for the rule to disregard all samples if $x>m$. If the score $x$ is an integer, e.g. $k$ or $(k+1)$, we can postulate an infinitude of values to which we can assign the probability $\alpha$ that $x>m$ in the range $k<m<k+1$.

It will be convenient to write $P(x>k)$ for the probability that $x$ exceeds $k$ and $P(x \geqslant k)=$ $P(x>k-1)$ for the probability that $x$ is not less then $k$. If $k$ is an integer there are $(s-k)$ score values in the range $x>k$ and $(s-k+1)$ in the range $x \geqslant k$, whence:

$P(x>k)=\frac{s-k}{s}$ and $P(x \geqslant k)=\frac{s-k+1}{s}$.

If $k+1>m \geqslant k$ so that $m$ is either an improper fraction in the interval between $k$ and $(k+1)$ or is the integer $k$ itself, we may write $m=(k+\varepsilon)$ and $k=(m-\varepsilon)$ for values of $\varepsilon$ in the range $0 \leqslant \varepsilon<1$.

When $\varepsilon=0$, we may write:

$P(x>m)=\frac{s-m}{s}$ and $P(x \geqslant m)=\frac{s-m+1}{s}$.

When $\varepsilon>0$,

$$
\begin{gathered}
P(x>m)=P(x>k)=\frac{s-k}{s}=\frac{s-m+\varepsilon}{s}, \\
P(x \geqslant m)=P(x \geqslant k)=\frac{s-k+1}{s}=\frac{s-m+1+\varepsilon}{s}, \\
\therefore P(x>m)>\frac{s-m}{s} \text { and } P(x \geqslant m)>\frac{s-m+1}{s} \ldots \text { (iv) }
\end{gathered}
$$

We may subsume both (iii) and (iv) to cover the possibility that $m$ may or may not be a whole number in the expressions:

$$
P(x>m) \geqslant \frac{s-m}{s} \text { and } P(x \geqslant m) \geqslant \frac{s-m+1}{s} .
$$

Let us now set $m=\alpha s$, so that:

$$
\begin{aligned}
& \text { Rule (i): } P(x>\alpha s) \geqslant 1-\alpha \quad \ldots \quad . \quad . . \\
& \text { Rule (ii): } P(x \geqslant \alpha s) \geqslant(1-\alpha)+\frac{1}{s}>(1-\alpha) . .
\end{aligned}
$$

The proportion of all samples whose score $x$ exceeds $\alpha s$ is thus no less than $100(1-\alpha)$ per cent.; and the proportion of all samples whose score $x$ is not less than $\alpha s$ is greater than $100(1-\alpha)$ per cent.
We may set out the implications of the foregoing statements as below:

\begin{tabular}{c|c|c|c|c}
\hline Event & $\begin{array}{c}\text { Probability } \\
\text { of its } \\
\text { Occurrence }\end{array}$ & $\begin{array}{c}\text { Equivalent } \\
\text { Assertion }\end{array}$ & $\begin{array}{c}\text { Probability } \\
\text { of its } \\
\text { Truth }\end{array}$ & $\begin{array}{c}\text { Probability } \\
\text { of its } \\
\text { Falsehood }\end{array}$ \\
\cline { 2 - 5 }$x>\alpha s$ & $\geqslant(1-\alpha)$ & $s<\frac{x}{\alpha}$ & $P_{t} \geqslant(1-\alpha)$ & $P_{f} \leqslant \alpha$ \\
$x \geqslant \alpha s$ & $>1-\alpha$ & $s \leqslant \frac{x}{\alpha}$ & $P_{t}>(1-\alpha)$ & $P_{f}<\alpha$ \\
\hline
\end{tabular}

We may express this by saying that our uncertainty safeguard for the assertion that $s$ is less than $20 x$ does not exceed 5 per cent. and our uncertainty safeguard for the assertion that $s$ is at least $20 x$ is

\begin{tabular}{|c|c|c|c|}
\hline Rule & $x=5$ & $x=10$ & $P_{f}$ \\
\hline $\begin{array}{l}\text { (i) } \\
\text { (ii) }\end{array}$ & $\begin{array}{l}s<100 \\
s<101\end{array}$ & $\begin{array}{l}s<200 \\
s<201\end{array}$ & $\begin{array}{l}\leqslant 0.05 \\
<0.05\end{array}$ \\
\hline
\end{tabular}
less than 5 per cent. On the basis of observations of single spins with scores of $x=5$ and $x=10$, respectively, our assertions would thus take the following form, if we deem $P_{f} \leqslant \alpha$ as an acceptable level of uncertainty:

To say that $s<100$ in this context is to say that the upper confidence limit is 99 . In terms of confidence limits we therefore write the above as:

\begin{tabular}{c|c|c|c}
\hline \multirow{2}{*}{ Rule } & \multicolumn{2}{|c|}{ Upper Confidence Limit of $s$} & \multirow{2}{*}{$P_{f}$} \\
\cline { 2 - 3 } & $x=5$ & $x=10$ & ${ }^{2}$ \\
\hline (i) & 99 & 199 & $\begin{array}{r}\leqslant 0.05 \\
<0.05\end{array}$ \\
\hline
\end{tabular}

Why we cannot express our confidence level in the form of an exact specification of the uncertainty safeguard of the form $P_{f}=\alpha$ will be clear if we state the foregoing rules in another way. In effect, Rule (i) signifies that we propose to disregard all samples if $x \leqslant \alpha s$, and Rule (ii) that we shall consistently disregard samples if $x<\alpha s$. We can get a backstage view of their implications, if we determine the proportion of excluded samples, i.e. the true uncertainty safeguard prescribed by each rule for values of $s$ in the neighbourhood of 200, when $\alpha=0.05$ defines the upper limit of acceptability for our uncertainty safeguard and the sample score is $x=10$. For $s=199,200$, and 201 respectively, $\alpha s=9 \cdot 95,10$, and 10.05 .

By Rule ( $i$ ) we disregard samples whose scores are 9,10 , and 10 . The exact probabilities $\left(P_{f}\right)$ of doing so are respectively:

$$
\frac{9}{199} ; \quad \frac{10}{200} ; \quad \frac{10}{201} .
$$


By Rule (ii) we disregard samples whose scores are 9,9 , and 10 with probabilities:

$$
\frac{9}{199} \quad ; \quad \frac{9}{200} ; \quad \frac{10}{201} \text {. }
$$

Thus the values of $P_{f}$ for $s$ in the neighbourhood of 200 are:

\begin{tabular}{c|c|c}
\hline$s$ & Rule $($ i $)$ & Rule $($ ii $)$ \\
\hline 199 & $\bumpeq 0.045$ & $\bumpeq 0.045$ \\
200 & 0.05 & 0.045 \\
201 & $\bumpeq 0.0497$ & 0.0497 \\
\end{tabular}

Rule (i) will make $P_{f}=0.05=\alpha$, when $s$ is an exact multiple of $20=\alpha^{-1}$; but otherwise $P_{f}<\alpha$. Rule (ii) makes $P_{f}$ nearly equal to $\alpha$, when $s$ is an exact multiple of 20 , but always less than $\alpha$.

We did not have to face the issue last discussed in the context of Model I $(b)$, because we invoked a normal approximation for the summation of the terms of a truly discrete binomial sample distribution. It is therefore instructive to re-examine the foregoing model situation on the assumption that the score $x$ is a continuous rectangular variate. We may then interpret $x \geqslant k$ as $x>\left(k-\frac{1}{2}\right)$ and $x \leqslant k$ as $x<\left(k+\frac{1}{2}\right)$. To accommodate all discrete values in the range $x=1$ to $x=s$ inclusive, we must accordingly extend the range of the continuous distribution from $x=\frac{1}{2}$ to $x=\left(s+\frac{1}{2}\right)$. On this understanding, our formal definition of the continuous rectangular distribution has merely to satisfy two conditions:

(a) the probability $f(x) d x$ that a score lies in the range $x \pm \frac{1}{2} d x$ is constant for all values of $x$, that is $f(x) d x=K . d x$;

(b) the complete integral is numerically equal to unity, that is

$$
K \int_{\frac{1}{2}}^{s+\frac{1}{2}} d x=1=K . s, \quad \text { and } \quad K=\frac{1}{s} .
$$

The probabilities that the score lies in the range from 1 to $k$ or beyond $k$ are then expressible as:

$$
\begin{aligned}
& \qquad \begin{aligned}
P(x \leqslant k) & =\frac{1}{s} \int_{\frac{1}{2}}^{k+\frac{1}{2}} d x=\frac{k}{s}, \\
\text { and } P(x>k) & =1-\frac{k}{s} .
\end{aligned}
\end{aligned}
$$

The above statement is exactly true of the discrete distribution, since $P(x \leqslant k)=P\left(x<k+\frac{1}{2}\right)$ if $x$ is necessarily an integer. In effect, we make our range from $\frac{1}{2} \Delta x$ to $s+\frac{1}{2} \Delta x$, since $\Delta x=1$; and we may neglect $\Delta x$ if $s$ is very large, as we must assume if we invoke the continuous distribution as a descriptive device. We shall then say that the range is from 0 to $s$, and admit fractional values of $x$ consistent with the specification:

$$
P(x>k)=\frac{1}{s} \int_{k}^{s} d x=1-\frac{k}{s} .
$$

Accordingly, we now proceed on the assumption that $x$ can have any real value in the range 0 to $s$. To make $P(x>k)=1-\alpha$ we then put $k=s \alpha$, so that:

$$
P(x>s \alpha)=1-\alpha .
$$

Within the framework of the rule implicit in the procedure, we then assign $(1-\alpha)$ as the probability of correctly asserting that

$$
s<\frac{x}{\alpha} .
$$

When $\alpha=0.05$, this is equivalent to assigning $P_{f}=0.05$ to the assertion that $s$ lies within the range from 1 to $20 x$.

We have hitherto confined our attention to a procedure which entitles us to assign to $s$ an upper confidence limit with an uncertainty safeguard $P_{f} \leqslant \alpha$. If we wish to place it with a pre-assigned uncertainty safeguard in an interval $a x>s>b x$, the form of statement we may make is no longer unique. If we may justifiably proceed on the assumption that we can assign an exact uncertainty safeguard $P_{f}=\gamma$ to what assertions we do make within the framework of a prescribed rule of procedure, i.e. that we may legitimately rely as above on the continuous distribution, we may write:

$$
P(k<x<m)=\frac{1}{s} \int_{k}^{m} d x=\frac{m-k}{s} .
$$

If we now write $k=\beta s$ and $m=\alpha s$,

$$
P(\beta s<x<\alpha s)=\alpha-\beta \text {. }
$$

We then assign an uncertainty safeguard $P_{f}=1-(\alpha-\beta)$ to the assertion:

$$
\beta>s>\frac{x}{\alpha} \text {. }
$$

If $\beta=0.025$ and $\alpha=0.975$ so that $P_{f}=0.05$, our final statement will thus be:

$$
40 x>s>\frac{40 x}{39} .
$$

Now $P_{f}=0.05$ if $\beta=0.01$ and $\alpha=0.96$. We are therefore entitled to assign $P_{f}=0.05$ as the uncertainty safeguard to the alternative assertion:

$$
100 x>s>\frac{25 x}{24} \text {. }
$$

When we write down $P(x>s \alpha)=1-\alpha$ or $P(\beta s<x<\alpha s)=\alpha-\beta$, we state the probability of an event, i.e. the value of the unit score $x$, within the framework of the classical theory of probability and the convenient fiction that the distribution is continuous. Our assertion signifies: for the fixed 
value $s$ of the relevant parameter, $P_{x . s}$ is the probability that the unit score will lie in such and such a range. We have refrained from writing the probability we assign to the equivalent assertions in the notation $P\left(s<x \alpha^{-1}\right)=1-\alpha$ or $P\left(\beta^{-1} x>s>\alpha^{-1} x\right)=\alpha-\beta$, lest we should hastily interpret them in terms of inverse probability, i.e. as if we could legitimately say: for the fixed value $x$ of the unit score, $P_{s . x}$ is the probability that $s$ will lie in the specified range. Such a form of words is inconsistent with Neyman's theory. We must interpret a statement in the form $P(a x>s>b x)=\gamma$ as a summary of the long run result of consistently adopting one and the same rule of conduct regardless of the value $(e . g . x=5)$ the score $x$ may have in any single trial, including the particular trial to which our specification of the interval estimate is referable. The formal statement of the rule will be adequate only if it explicitly specifies $x$ as an unknown which may assume any value within its admissible range. We misinterpret it if we condense our verdict in such a form as:

$$
P\left(200>s>\frac{200}{39}\right)=0.95 \text {. }
$$

This is an act of self-deception into which we easily slide, if we write the formal identities:

$$
\begin{gathered}
\beta(h+d h)=x=\alpha h, \\
\frac{x}{h}-\frac{x}{h+d h}=\alpha-\beta=\frac{x \cdot d h}{h(h+d h)}, \\
P(h+d h>s>h)=\frac{x \cdot d h}{h^{2}} .
\end{gathered}
$$

We have now eliminated any reference to $x$ as a variable in the expression on the left, and have obtained on the right what is seemingly the element of a probability distribution and satisfies the fundamental property of the latter, if we fix $x$ and define the range of $s$ from $h=x$ to $h=\infty$, so that,

$$
x \int_{x}^{\infty} h^{-2} \cdot d h=1
$$

This step, which leads to what Fisher calls a fiducial probability distribution, is admissible only if we can legitimately confine our statements to situations in which $x$ has one and the same value $(e . g . x=5)$. We could then write:

$$
P(s<k)=x \int_{x}^{k} h^{-2} \cdot d h=\frac{k-x}{k}
$$

If $k=20 x$, we thus obtain by a somewhat circuitous route a result already derived within the framework of the assumed continuous rectangular distribution, i.e. $P(s<20 x)=0 \cdot 95$. It follows that many results embodied in Fisher's approach to interval estimation will tally with those to which the theory of confidence intervals leads us; and indeed many statisticians were at one time blind to what we now see to be a radical difference. If we conceive $x . f(h) d h$ as an element of a probability distribution, we have to regard $h$ and $x$ as independent to arrive at a numerical result consistent with confidence theory in the continuous domain; but we can do so only if we then treat $x$ as a constant in the algebraic manipulation. We thus implicitly fix our interval in terms of a pre-assigned value of $x$ to arrive at the specification of a probability dependent thereon; but this is inconsistent with the programme of Neyman's theory, which specifies the interval in terms of a pre-assigned probability independent of the outcome of any single trial and hence of any pre-assigned value of $x$.

\section{Relation of Estimation to Test Procedure}

If we regard the problem of estimation as that of assigning a probability to the truth of the assertion that some unique definitive parameter of a homogeneous universe lies between specified limits, we sidestep the disquieting dilemma with which the balance sheet of Bayes confronts us. Bayes's theorem is essentially about a stratified universe, e.g. a bag in which some pennies with unlike faces are unbiassed and one penny (through a defect of minting) has the King's head on both sides. In effect, it says:

"To know how often I should be right in judging a coin taken from the bag to be the one defective coin after getting ten successive heads in a single 10-fold toss, I must also know how many other coins the bag contains."

If we presume its relevance to a general theory of test procedure, one horn of the dilemma to which the theorem draws attention is that we rarely have such knowledge. The other is that all the coins may indeed be alike, - and our only source of relevant information is the one coin we have tossed. The theory of confidence intervals sidesteps the dilemma by restricting our attention to all we can know in situations which disclose prior knowledge of neither sort. It is the writers' belief that Neyman (1934) did not overstate the novelty or the importance of the viewpoint we have explored against the background of the preceding models, when he declared:

The solution of the problem which I described as confidence intervals has been sought by the greatest minds since the work of Bayes 150 years ago. Any recent book on the theory of probability includes large sections concerning this problem. The present solution means, I think, not less than a revolution in the theory of statistics. 
The model situations we shall examine below suggest an approach, alternative to and more sophisticated than that of the foregoing section, to clarify what is common to the domain of test procedure and to the domain of estimation. They are also of subsidiary interest inasmuch as they sidestep the Bayes's dilemma by a route superficially different from that we have so far followed. If we now explore their properties on that understanding, it is not because we believe Bayes's theorem to have any necessary relevance to a theory of interval estimation. We shall examine the consequences of the assumption that it may have, only because of a widely prevalent belief that any adequate theory of statistical decision must come to terms with the concept of prior probability.

In our previous communication, we examined a laboratory situation which precisely recalls the model appropriate to the issue Bayes propounds. We postulate a Drosophila culture known to contain two sorts of female flies, some with a sex-linked lethal gene and others normal. Our problem is to attach an uncertainty safeguard $\left(P_{f}\right)$ to a decision in favour of the hypothesis that a particular female fruitfly is of one or the other sort. In this set-up each hypothesis is referable to an existent subpopulation at risk; and we can speak about the prior probability assignable to a hypothesis without danger of self deception. It is meaningful to do so, because we conceive the situation as one which offers us a tangible preliminary choice at random, i.e. the extraction of the particular fly from the culture so constituted; but the choice is indeed tangible only because we initially possess the information that the culture contains two sorts of flies. It would not be a real choice if we had to make the decision on the understanding that the females are of one sort only. In any acceptable sense of the term, the prior probability of one hypothesis is then zero and that of the other is unity. If we could correctly assign the appropriate value to each hypothesis there would be no problem to solve.

To those whose approach to problems of cognition is essentially behaviouristic, it is therefore by no means obvious that the model situation appropriate to Bayes's theorem has any relevance to circumstances in which we have no opportunity of exercising the preliminary act of choice prescribed thereby; but there need be no dispute about the relevance of the prior probabilities to the prescription of a test procedure. Our examination of the mixed culture situtation shows that neither ignorance of the precise prior probabilities each referable to an existent population nor the unreality of the assumption that we necessarily carry out the enquiry in two stages need deter us from formulating a rule of decision with an assignable uncertainty safeguard. When we choose our rejection criterion to make the error of the first kind equal to the error of the second kind $(\alpha=\beta)$, we arrive at the identity $P_{f}=\alpha$ for all values of the prior probabilities, and the relation $\alpha \leqslant P_{f} \leqslant \beta$ for $\beta>\alpha$ is likewise true for all values of the prior probabilities, including the limiting case when they are respectively zero and unity. Thus the rule holds good, whether we can realistically interpret the decision against the background of Bayes's model or in situations to which the two-stage sampling procedure implicit in the model has no factual relevance.

This is the course we now propose to adopt with respect to interval estimation. Our new models will admit of a factual preliminary choice of the sub-universe from which we sample, with a view to exhibiting the irrelevance of such an assumption and its implications to the procedure of interval estimation. Indeed, we shall postulate situations to which the Bayes balance sheet is truly relevant. Our universe will be a stratified universe, and our problem to attach an acceptable uncertainty safeguard to the assertion that a parameter definitive of the particular stratum from which we take a particular sample lies within a specified range.

Model II $(a)$.-With this end in view, we shall suppose that someone spins forty times one of 100 lottery wheels chosen at random, recording the mean score $\left(M_{x}\right)$. Each such wheel has 1,024 sectors like the wheel of MODEL I $(a)$ with scores of $x,(x+1),(x+2) \ldots$ $(x+9),(x+10)$, allocated respectively to $1,10,45 \ldots$ 10,1 sectors. We do not know the value of $x$ associated with the particular wheel selected for the spin; but we do know however that each wheel is one of eleven types as follows:

\begin{tabular}{c|c|c}
\hline Type & No. of Wheels & Value of $x$ \\
\hline II & 1 & $0 \cdot 5$ \\
III & 3 & $0 \cdot 6$ \\
IV & 10 & $0 \cdot 7$ \\
V & 17 & $0 \cdot 8$ \\
VI & 20 & $1 \cdot 1$ \\
VII & 7 & $1 \cdot 3$ \\
VIII & 12 & 1.5 \\
IX & 3 & 1.8 \\
X & 8 & 1.9 \\
XI & 2 & $2 \cdot 0$ \\
& 17 & $2 \cdot 1$ \\
\hline
\end{tabular}

In this model set-up, we may construct eleven admissible hypotheses about the value of $x$, and hence of the expected mean $M=(x+5)$. For each hypothesis, the standard deviation of the distribution of the observed mean $\left(M_{x}\right)$ of the 40-fold spin is $\sigma_{m}=0 \cdot 25$, and to each hypothesis we can assign a prior probability in Bayes's sense. If the observed mean score for the 40 -fold spin is $6 \cdot 3$, as for MODEL I $(a)$, the relevant information is as follows: 


\begin{tabular}{c|c|c|c}
\hline Hypothesis & Prior Probability & $M$ & $(M-M x) \div \sigma m$ \\
\cline { 2 - 3 } II & 0.01 & 5.5 & -3.2 \\
III & 0.03 & 5.6 & -2.8 \\
IV & 0.10 & 5.7 & -2.4 \\
V & 0.17 & 5.8 & -2.0 \\
VI & 0.20 & 6.1 & -0.8 \\
VII & 0.07 & 6.3 & 0 \\
VIII & 0.12 & 6.5 & +0.8 \\
IX & 0.03 & 6.8 & +2.0 \\
X & 0.08 & 6.9 & +2.4 \\
\hline
\end{tabular}

We shall now make the following rule. We shall reject some hypotheses as inadmissible and reserve judgment on others which we shall accordingly regard as admissible, applying to each hypothesis the same criterion of rejection, i.e. that it assigns to the deviation of the observed score $\left(M_{x}=6 \cdot 3\right)$ from the expected value $(M)$ prescribed by the particular hypothesis a value numerically greater than $2 \sigma_{m}$. We then reject all hypotheses, except IV-VIII inclusive, and are left with the assertion that $M$ lies in the range 5.8-6.8, corresponding to values of $x$ from 0.8 to $1 \cdot 8$.

Our uncertainty safeguard for the rejection of every hypothesis when true is $\alpha=0.05$ since our rejection criterion is modular. That the unconditional uncertainty safeguard for the final verdict is also 0.05 , as for MoDEL I (a), we may make explicit as follows. We first remind ourselves that we can falsely reject only one hypothesis since only one can be true. Thus the unconditional probability of a false verdict is the unconditional probability of falsely rejecting one or other of an exclusive set of hypotheses, and is therefore obtainable by recourse to the addition rule. If $\boldsymbol{P}_{\boldsymbol{h}}$ is the prior probability that the particular hypothesis $H$ is applicable to the situation, i.e. that we choose at random a wheel of type $H$ to spin, the probability of falsely rejecting it is $\alpha P_{h}$; and by definition:

$$
\sum_{h=1}^{h=11} P_{h}=1 .
$$

The probability of making a false decision is the probability of falsely rejecting any one of the hypotheses, i.e.:

$$
\sum_{h=1}^{h=11} P_{h} \cdot \alpha=\alpha \sum_{h=1}^{h=11} P_{h}=\alpha .
$$

Thus $\alpha$ is our uncertainty safeguard to the assertion that $M$ lies within the prescribed limits; and the prior probabilities of Bayes do not affect its value. We have thus arrived at exactly the same result as in the MoDEL I (a) situation, where we set the same uncertainty safeguard to the same range of admissible values of the parameter $x$ in the unstratified universe of one and the same wheel.

In the set-up of this Model, we regard any one of a limitless number of values $p$ may have as a hypothesis referable to a conceivably, but not necessarily, existent population at risk. We thus interpret the process of estimation as a method of screening an exhaustive set of hypotheses as admissible or otherwise by successively applying to each a test prescribing the same probability of rejection if the hypothesis is indeed true. Our universe of hypotheses so conceived is a stratified universe, in which strata with the same definitive parameter $\boldsymbol{P}_{h}$ provisionally constitute an existent population at risk with an assignable finite prior probability in the jargon of Bayes's theorem. Bayes's prior probabilities $\left(P_{h}\right)$ are then inherent in the initial formulation of the problem; but they do not appear in the solution. Consequently, we are free to assign to the prior probability of any single hypothesis any value in the range 0 to 1 consistent with the restriction that the sum of all the prior probabilities is unity. Whether there corresponds an existent population to a particular hypothesis in our fictitious stratified universe is therefore immaterial. That a particular hypothesis to which we apply the test corresponds to no existent population merely means that $\boldsymbol{P}_{\boldsymbol{h}}=\mathbf{0}$. To conceive the universe as unstratified is to assign $P_{h}=1$ to one stratum and $P_{h}=0$ to every other one. In this sense, MODEL $I$ is therefore a limiting case of MODEL II.

This way of looking at the problem of estimation makes the distinction between the domain of test decision and estimation less clear-cut than the alternative. If we interpret the procedure of estimation in terms of the model of this section, we can regard it as the performance of a battery of tests, but the score value which defines the criterion of rejection is different for each test and the decision to reject any one hypothesis or group of hypotheses does not prescribe acceptance of any other single hypothesis. We successively apply to each a test involving a new value of the score deviation $(x-M)$ as the criterion which ensures the same probability of rejection for each hypothesis when true. If we assert that one group of hypotheses constitutes an admissible (in contradistinction to a residual group as an inadmissible) set, we then do so on the assumption that one of the former is identifiable with the correct one.

MODEL II (c).-In our choice of a common criterion of rejection for the hypotheses sifted in the treatment of the foregoing model, we may assume, as we have assumed, a normal distribution of the mean score without incurring exceptionable error. Accordingly, we have defined the uncertainty safeguard of the prescribed rule by the identity $P_{f}=\alpha$; but any such formulation is strictly valid only in the fictitious domain of the continuous variate. It will therefore be profitable to examine a model situation in which we cannot legitimately invoke the normal, or any other continuous, distribution.

In the homogeneous universe of MODEL I, we have seen that we can set an upper limit $\left(P_{f}<\alpha\right.$ or $\left.P_{f} \leqslant \alpha\right)$ to the uncertainty safeguard we attach to a confidence boundary in the domain of discrete score values; but 
we cannot make an exact statement of the form $P_{f}=\alpha$. Let us now therefore look at the problem raised by MODEL I (c) of SECTION 2 (above) as one of sampling in a stratified universe. We shall postulate as below an assemblage of one hundred lottery wheels of twelve types with consecutive scores 1 to $m$ inclusive, if $s=m$ is the number of sectors of a wheel of type $H$. Thus we have twelve hypotheses about $s$ to explore, each referable to an existent population at risk; and we shall once more limit our decisions to rejection and reservation of judgment. We know the score $x$ of a single spin without knowing the type of wheel to which it is referable. Our problem will be to assign a probability to an admissible set of hypotheses.

\begin{tabular}{c|c|c|c}
\hline $\begin{array}{c}\text { Type of } \\
\text { Wheel } \\
(H)\end{array}$ & $\begin{array}{c}\text { No. of } \\
\text { Sectors } \\
\left(s_{h}\right)\end{array}$ & $\begin{array}{c}\text { No. of } \\
\text { Corresponding } \\
\text { Wheels }\left(N_{h}\right)\end{array}$ & $\begin{array}{c}\text { Prior Probability } \\
\text { of Choice } \\
\left(P_{h}=\frac{N_{h}}{100}\right)\end{array}$ \\
\hline 1 & 5 & 13 & $0 \cdot 13$ \\
2 & 19 & 2 & $0 \cdot 02$ \\
3 & 20 & 1 & $0 \cdot 01$ \\
4 & 21 & 7 & $0 \cdot 03$ \\
5 & 39 & 12 & $0 \cdot 07$ \\
6 & 40 & 3 & $0 \cdot 12$ \\
7 & 99 & 4 & $0 \cdot 03$ \\
8 & 100 & 10 & $0 \cdot 04$ \\
9 & 101 & 15 & $0 \cdot 09$ \\
10 & 199 & 200 & $0 \cdot 15$ \\
11 & 201 & 100 & $0 \cdot 21$ \\
\hline 12 & Total & 100 & $1 \cdot 00$ \\
\hline
\end{tabular}

For MODEL I (c) we formulated two rules:

$$
\begin{aligned}
& \text { Rule (i): } s<\frac{x}{\alpha} \text { with } P_{f} \leqslant \alpha \\
& \text { Rule (ii): } s \leqslant \frac{x}{\alpha} \text { with } P_{f}<\alpha
\end{aligned}
$$

In effect, the first rule states that we reject the hypothesis $s=s_{h}$ unless $x>\alpha s_{h}$; and the second states that we reject the hypothesis $s=s_{h}$ unless $x \geqslant \alpha s_{h}$. Thus our rejection criteria are:

Rule (i): Reject if $x \leqslant \alpha$ sh with $P_{f} \leqslant \alpha$

\begin{tabular}{|c|c|c|c|c|c|c|}
\hline \multirow{2}{*}{$\begin{array}{c}\text { Hypo- } \\
\text { thesis } \\
(h)\end{array}$} & \multirow{2}{*}{$\begin{array}{c}\text { No. of } \\
\text { Sectors } \\
\left(s_{h}\right)\end{array}$} & \multirow{2}{*}{$\left|\begin{array}{c}\text { Cri- } \\
\text { terion } \\
\left(\alpha s_{h}=\right. \\
\left.0 \cdot 05 s_{h}\right)\end{array}\right|$} & \multicolumn{2}{|c|}{$x=5$} & \multicolumn{2}{|c|}{$x=10$} \\
\hline & & & $\begin{array}{c}\text { Verdict } \\
\text { by } \\
\text { Rule }(i)\end{array}$ & $\begin{array}{c}\text { Verdict } \\
\text { by } \\
\text { Rule (ii) }\end{array}$ & $\begin{array}{c}\text { Verdict } \\
\text { by } \\
\text { Rule }(i)\end{array}$ & $\begin{array}{l}\text { Verdict } \\
\text { by } \\
\text { Rule (ii) }\end{array}$ \\
\hline 1 & 5 & $0 \cdot 25$ & Open & Open & Open & Open \\
\hline 2 & 19 & 0.95 & Open & Open & Open & Open \\
\hline 3 & 20 & $1 \cdot 00$ & Open & Open & Open & Open \\
\hline 4 & 21 & 1.05 & Open & Open & Open & Open \\
\hline 5 & 39 & $1 \cdot 95$ & Open & Open & Open & Open \\
\hline 6 & 40 & $2 \cdot 00$ & Open & Open & Open & Open \\
\hline 7 & 99 & $4 \cdot 95$ & Open & Open & Open & Open \\
\hline 8 & 100 & $5 \cdot 00$ & REJECT & Open & Open & Open \\
\hline 9 & 101 & $5 \cdot 05$ & REJECT & REJECT & Open & Open \\
\hline 10 & 199 & $9 \cdot 95$ & REJECT & REJECT & Open & Open \\
\hline 11 & 200 & $10 \cdot 00$ & REJECT & REJECT & REJECT & Open \\
\hline 12 & 201 & $10 \cdot 05$ & REJECT & REJECT & REJECT & REJECT \\
\hline
\end{tabular}

Rule (ii): Reject if $x<\alpha$ sh with $P_{f}<\alpha$
As below, we may then draw up a table of verdicts based on each of the foregoing rules for different experiments in which $x=5$ and $x=10$ respectively. In each case we assume that $\alpha=0.05$ is an acceptable level of uncertainty.

The range of $s$ values covered by open verdicts thus corresponds precisely with the outcome of our examination of MODEL I (c) for which the upper confidence limits are 99 and 199 respectively for $x=5$ and $x=10$ with $P_{f} \leqslant 0.05$ (Rule (i)), or 100 and 200 respectively for $x=5$ and $x=10$ with $P_{f}<0.05$ (Rule (ii)). The meaning of the correspondence is evident if we recall the meaning of the true conditional uncertainty safeguard $\left(P_{f, h}\right)$ of hypothesis $H$ in the domain of discrete score values. If our criterion of rejection is $x \leqslant \alpha s$, we exclude only samples whose score value is $x=\alpha s$ when $\alpha s$ itself is in integer. Thus $P_{f . h}$, the proportion of excluded score values when hypothesis $H$ is true, is the ratio to $s$ of the nearest integer not exceeding $s$ and is always less than or equal to $\alpha$. If $0 \leqslant \varepsilon_{h}<1$ we may thus write:

$$
\begin{aligned}
P_{f . h} & =\alpha-\varepsilon_{h}, \\
\therefore P_{f}=\sum_{h=1}^{h=12} P_{h} . P_{f . h} & =\alpha \sum_{h=1}^{h=12} P_{h}-\sum_{h=1}^{h=12} P_{h} . \varepsilon_{h}, \\
\therefore P_{f} & =\alpha-\sum_{h=1}^{h=12} P_{h} . \varepsilon_{h} .
\end{aligned}
$$

Since we have chosen the rejection criterion so that $P_{f . h} \leqslant \alpha$, all values of $\varepsilon_{h}$ must be zero or positive. Rule (ii) asserts that they are all positive, whence we obtain, as for MoDEL I (c),

$$
P_{f}<\alpha \text {. }
$$

In this instance, some values of $\varepsilon_{h}$ are positive when we apply Rule $(i)$ and others zero. Thus $P_{f}<\alpha$ as before; but this is not inconsistent with the assertion $P \leqslant \alpha$, being included therein. A generalized MODEL II situation must take stock of the possibility that $P_{f . h}=\alpha$ for each wheel as would be true if we knew that the recorded score referred to a wheel of any one of types $3,6,8,11$ above. For each of these $P_{f . h}=0.05$ and $\varepsilon_{h}=0$, as will be seen by citing the values of $P_{f . h}$ prescribed by our rejection criterion, viz.:

\begin{tabular}{c|c|c|c}
\hline$s_{h}$ & $\alpha s_{h}$ & Rule $(i)$ & Rule $(i i)$ \\
\hline 5 & 0.25 & 0.0000 & 0.0000 \\
19 & 0.95 & 0.0000 & 0.0000 \\
20 & 1.00 & 0.0500 & 0.0000 \\
21 & 1.05 & 0.0476 & 0.0476 \\
39 & 1.95 & 0.0256 & 0.0256 \\
40 & 2.00 & 0.0500 & 0.0250 \\
99 & 4.95 & 0.0404 & 0.0404 \\
100 & 5.00 & 0.0500 & 0.0404 \\
101 & 5.05 & 0.0495 & 0.0495 \\
199 & 9.95 & 0.0452 & 0.0452 \\
200 & 10.00 & 0.0500 & 0.0450 \\
201 & 10.05 & 0.0497 & 0.0497 \\
& & & \\
\hline
\end{tabular}


In the treatment of ModeL I (c) we have already recognized one reason for regarding the concept of fiducial probability as an inadequate basis for a theory of statistical inference in that it restricts the field of discussion to continuous variates. Further consideration of the model situation we have last discussed gives us an opportunity for contrasting two theories of interval estimation from a different viewpoint.

Fiducial probability takes its origin in concepts, some of which are common to the theory of confidence intervals; but Neyman's development of the latter is inconsistent with Fisher's interpretation of the former, unless there is some sense in which only one admissible pre-assigned rule of test procedure is appropriate to one and the same situation. Models I (c) and II (c) do indeed refer to a situation in which only one such rule invites our attention as relevant to the end in view; but we have not excluded the possibility that more than one might each have seemingly equal claims to commend it from a purely formal viewpoint. We shall now examine a situation in which this dilemma arises.

Since a continuum is implicit in the concept of fiducial probability, we shall postulate a continuous rectangular distribution over the range $\frac{1}{2}$ to $s+\frac{1}{2}$, and examine what statements we may make when we draw two unit samples with scores $x_{1}$ and $x_{2}$. Two, though not the only two, rules which we may formulate will serve our purpose well enough for heuristic purposes. We shall alternatively seek to prescribe an upper confidence limit to $s$ with an uncertainty safeguard $\alpha$ by recourse to:

(i) the maximum score $x_{m}$ being $x_{m}=x_{1}$ if $x_{1} \geqslant x_{2}$, and $x_{m}=x_{2}$ if $x_{2} \geqslant x_{1}$

(ii) the score sum $x_{12}=x_{1}+x_{2}$.

The probability that $x_{m} \leqslant m$ is the probability assignable to the joint occurrence that each score lies in the range from $x=0$ to $x=m$ inclusive, i.e.:

$$
P\left(x_{m} \leqslant m\right)=\frac{m^{2}}{s^{2}} \text { and } P\left(x_{m}>m\right)=1-\frac{m^{2}}{s^{2}}
$$

We wish our final assertion to take the form $s<k x$ with a probability $(1-\alpha)$ of correct assertion if we consistently follow the test procedure, whence we write:

$$
\begin{aligned}
P\left(x_{m}>m\right) & =(1-\alpha) \frac{m^{2}}{s^{2}}=\alpha \quad \text { and } \\
P\left(x_{m}>s \sqrt{\alpha}\right) & =1-\alpha .
\end{aligned}
$$

Within the framework of this rule, we then assign $\alpha$ as the uncertainty safeguard to the assertion:

$$
s<\frac{x_{m}}{\sqrt{\alpha}}
$$

If we base our test procedure on $x_{12}$ defined as above, the reader unfamiliar with the continuous rectangular distribution will find it helpful first to make a simple chessboard diagram of the 2 -fold discrete score sum distribution. It is then evident that we may express the probability that $x_{12}$ lies in the range 2 to $k$ if $x=1$ is the origin of the unit score distribution in two ways:

$$
\begin{array}{ll}
P\left(x_{12}>k\right)=\frac{(2 s-k)(2 s-k+1)}{2 s^{2}} & \text { when } k>s+1 ; \\
P\left(x_{12}>k\right)=\frac{1-k(k-1)}{2 s^{2}} \quad \text { when } k \leqslant s+1 .
\end{array}
$$

For the continuous case we may represent our chessboard geometrically as a rectangle of area $s^{2}$ and the region in which all values $x_{12} \leqslant k$ lie when $k \leqslant s$ as a triangle of area $\frac{1}{2} k^{2}$. Since we wish to associate a probability $(1-\alpha)$ near unity to the truth of the assertion $s<k^{-1} . x$, our concern will be with the smaller value of $k$ (Fig. 3).
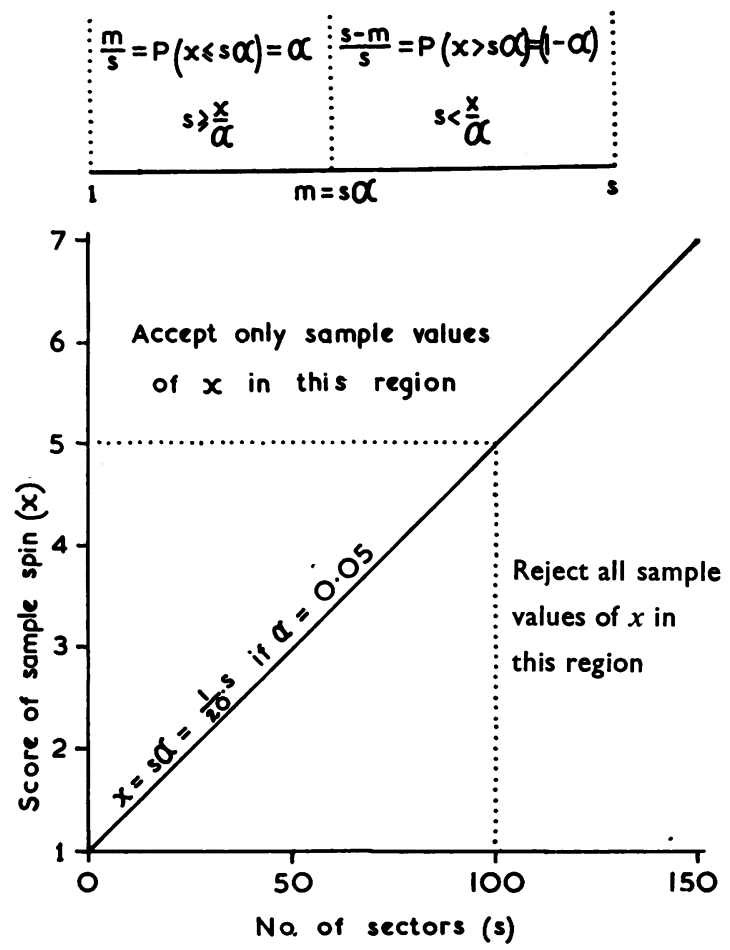

FIG. 3.-Graphical representation of one-sided confidence limit for the number of score classes (s) of a rectangular universe when the score $x$ refers to a single sample.

For the continuous case we then write:

$$
\begin{gathered}
P\left(x_{12}>k\right)=1-\frac{k^{2}}{2 s^{2}}=1-\alpha, \\
\therefore P\left(x_{12}>s \sqrt{2} \alpha\right)=1-\alpha .
\end{gathered}
$$


Our second rule thus assigns $\alpha$ as the uncertainty safeguard to the assertion

$$
s<\sqrt{\frac{x_{12}}{2 \alpha}}
$$

We thus have two rules which assign different values to the upper confidence limit of $s$ at one and the same confidence level $(1-\alpha)$. In the strictly behaviourist formulation of confidence theory by Neyman this involves no inconsistency. One rule may seem better than another, if it incorporates more "information"; but its use may have drawbacks which outweigh its merit on that account. In Fisher's theory of interval estimation no such freedom of choice is admissible. The avowed intention of the concept of fiducial probability is to express the intensity of legitimate conviction referable to a particular sample. If so, only one rule can be right, namely the rule which invokes all the information the sample supplies. Fisher speaks of a statistic, i.e. sample score, which has this property, as the sufficient one.

The two statistics $x_{m}$ and $x_{12}$ used in the foregoing situation will serve to illustrate what is and what is not a sufficient statistic if we now consider $x_{1}$ and $x_{2}$ as unit samples from a discrete rectangular universe with a range of scores from 1 to $s$ inclusive. In deriving a rule on the basis of either we have suppressed any explicit specification of $x_{1}$ and $x_{2}$. If our chosen statistic is defective it can be so only for that reason. We shall therefore ask: have we lost anything by withholding such information? We may answer this by considering the consequences of confining our attention in a sequence of trials to samples with some pre-assigned value of $x_{m}$ or $x_{12}$.

Let us first suppose the pre-assigned value of $x_{m}$ to be 3 . The different sorts of double samples that are consistent with this value occur with equal frequencies and are specifiable as follows: $(1,3),(2,3)$, $(3,3),(3,2),(3,1)$. This set of equally frequent values is the same for all values of $s$ consistent with the specification $x_{m}=3$. Thus we have suppressed no information about $s$ by scoring our sample in this way. Is the same true of $x_{12}$ ?

Let us now consider samples with reference to which $x_{12}=8$. This specification is consistent with any value $s \geqslant 4$, but this condition does not suffice to specify what individual values $x_{1}$ and $x_{2}$ have. If $s=4$, the only double sample consistent with the specification $x_{12}=8$ is $(4,4)$. If $s=5$, three paired score values are allowable: $(3,5)$, $(4,4),(5,3)$. If $s=6$ we may have: $(2,6),(3,5)$, $(4,4),(5,3),(6,2)$. Thus we can say more about $s$, if we know the individual score of $x_{1}$ and $x_{2}$ than we can if we know only the value of the insufficient statistic $x_{12}$; but the individual values of $x_{1}$ and $x_{2}$ tell us no more than we already know, if told the value of the sufficient statistic $x_{m}$.

We have now to state the definition of a sufficient statistic formally. To do so, we first remind ourselves that to each 2-fold sample specified in terms of the sequence of unit samples we may assign as above a bivariate score, e.g. $(3,5)$ or $(5,3)$. We may then speak of $P_{12 . s}$ as the unconditional probability that any sample has the bivariate score $\left(x_{1}, x_{2}\right)$ and $P_{12 . m}$ as the conditional probability that it has this score if $x_{m}$ is the maximum score. In the same sense, we may label the unconditional probability of a multivariate score $\left(x_{1}, x_{2}, x_{3} \ldots x_{r}\right)$ definitive of an $r$-fold sample as $\left.P_{(1.2 .3} \ldots r\right) . p$ for a distribution whose definitive parameter is $p$ and $\left.P_{(1,2,3} \ldots\right) r . x$ as its conditional probability when the sample statistic is $x$, if we can define it from our knowledge of $x$ alone. We may then define by $P_{x, p}$ the probability that the sample statistic will be $x$ if the parameter is $p$ and obtain by recourse to the product rule:

$$
P_{(1,2 \ldots, r) . p}=P_{x, p} . P_{(1,2 \ldots r) \cdot x}
$$

We have now split into two factors the unconditional probability of getting the multivariate score which summarizes all the information the sample supplies, since its specification incorporates both the numerical values of each constituent unit sample and the order in which they turn up. One of these factors is independent of $p$ if the statistic is sufficient, i.e. if (as is true of $P_{12 . m}$ ) we can specify it without knowing the value of the universe parameter. We thus take as our formal criterion of a sufficient statistic the resolution of the probability of the multivariate score into two factors of which one does not contain $p$.

By recourse to a simple chessboard lay-out of $s^{2}$ cells, with border scores from $x=1$ to $x=s$ inclusive and $x=1$ to $x=s$ inclusive, we may amplify this breakdown with reference to $x_{m}$ and $x_{12}$ for the discrete rectangular universe. Each cell of the grid is referable to a unique pair of values $x=x_{1}$ and $x=x_{2}$, but the same value of $t=\left(x_{1}+x_{2}\right)$ or of $x_{m}=m$ is assignable to more than one cell if $t=2$. Cells specified by $x_{m}=m$ lie on two sides of a square of $m$ cells, there being $(2 m-1)$ in all. If we write $P_{12 . s}$ for the probability that the sample records the unique pair of score values $x_{1}$ and $x_{2}$ when the number of sectors is $s$, and $P_{12 . m}$ for the probability that $x_{m}=m$ when $x$ has these two values on the same assumption, and $P_{m . s}$ for the probability that $x_{m}=m$ when $s$ is the number of sectors, we thus see that

$P_{12 . s}=\frac{1}{s^{2}} ; \quad P_{12 \cdot m}=\frac{1}{2 m-1} ; \quad P_{m . s}=\frac{2 m-1}{s^{2}}$. 
Hence, in accordance with the product rule for conditional probabilities,

$$
P_{12 . s}=P_{12 . m} . P_{m . s} \text {. }
$$

We have thus split the probability assignable to the bivariate score $x_{1}, x_{2}$ into two factors, one of which $\left(P_{12} . m\right)$ is independent of $s$; and we might be tempted to think that we could specify a corresponding identity $P_{12 . s}=P_{12 . t} . P_{t . s}$, referable to the probabilities of getting the score sum $t$ when there are $s$ factors and getting the particular value of the bivariate score if also $\left(x_{1}+x_{2}\right)$ has the particular value $t$. Actually we cannot do so. All samples such that $\left(x_{1}+x_{2}\right)=t$ lie in a diagonal of $(t-1)$ cells if $s \geqslant(t-1)$; and if we knew this we might write $P_{12 . t}=(t-1)^{-1}$, which is again independent of $s$. Thus, if $s \geqslant 4$, there will be four cells in the diagonal corresponding to $t=5$; but there will be only two cells in it if $s=3$. Given $t$, we can say that $s \geqslant \frac{1}{2} t$, for example $s>2$ if $t=5$, but we cannot say that $s \geqslant(t-1)$. The mere fact that $t=5$ is therefore insufficient to assign a unique value to the conditional probability $P_{12 . t}$.

In the same sense we may speak of the number $(x)$ of successes in an $r$-fold sample from an infinite two-class universe as a sufficient statistic of the parameter $p$. We may denote by $P_{(1 \cdot 2 \cdot 3 \ldots r) p}$ the probability that the sample records successes and failures in a fixed order, there being $r_{(x)}$ different samples so distinguishable for the particular value $x$. Thus we may write:

$$
\begin{aligned}
\left.P_{(123} \ldots r\right) \cdot p & =p^{x} \cdot q^{x} ; \\
\left.P_{(123} \ldots r\right) \cdot x & =\frac{1}{r_{(x)}} ; \\
P_{x \cdot p} & =r_{(x)} p^{x} \cdot q^{x} \\
\left.P_{(123} \ldots r\right) \cdot p & \left.=P_{(123} \ldots r\right) \cdot x \cdot P_{x, p} .
\end{aligned}
$$

One circumstance which gives the concept of sufficiency a peculiar importance vis à vis Fisher's approach to the problem of interval estimation is that it is not always possible to specify a sample by a statistic which is sufficient in his sense of the term. Since the fiducial probability distribution is in his formulation referable only to sufficient statistics, and only to sufficient statistics themselves referable to continuous distributions, the fiducial theory of interval estimation is of much more limited application on its own terms than is Neyman's theory of confidence intervals.*

${ }^{*}$ The following citation specifies the attitude of Fisher (1936) attitude to the concept of sufficiency:

which of This consideration is vital to the fiducial type of argument, which purports to infer exact statements of the probabilities that unknown hypothetical quantities, or that future observations, shall lie within assigned limits, on the basis of a body of observational experience. No such process could be justified unless the relevant information latent in this experience were exhaustively mobilised and incorporated in our inference.
Neyman's preference for the expression inductive behaviour, in contradistinction to the traditional term inductive inference, forces on our attention a cleavage which admits of no compromise. It gets into focus an issue which must ultimately dictate our attitude to the place of statistical theory in scientific enquiry. It is not merely a revision of algebraic techniques or a matter of concern for the professional mathematician as such. It invites us to undertake a reorientation of our mental habits on a different plane. If we decide to adopt a consistently behaviourist viewpoint, the consequences will indeed be far more drastic than most of our contemporaries as yet foresee.

\section{INTERVAL Estimation OF DifferenCES}

For reasons already set forth we assume that a prophylactic or therapeutic trial has as its end in view to assess what advantage will accrue from the substitution of one treatment, Treatment $(B)$, for another elsewhere referred to as the yardstick treatment, Treatment $(A)$. Since the customary null hypothesis procedure takes no cognizance of the operational intention of the trial, it is not unimportant to make this explicit; and since alternative and more recently prescribed statistical procedures presuppose definition of a measure of relative efficacy, it is not trivial to be equally explicit about the measure we prefer to use. When the method of scoring treatment efficacy is taxonomic, as defined in the previous communication, we shall here assume provisionally that our main concern will be the additional number of cases likely to benefit from the substitution of Treatment $B$ for Treatment $A$; and the unequivocal measure of this advantage is the difference between the parameters $p_{b}$ and $p_{a}$ definitive of proportionate success in the putative universes of which the treatment groups are respectively samples. When the method of scoring is representative, our corresponding criterion of operation advantage will be the absolute difference between the corresponding true mean scores, if the latter are expressible (e.g. duration of stay in hospital) in terms directly relevant to costing or humane considerations. Otherwise, as when the score is referable to a laboratory test, the crude difference (in contradistinction to a ratio or other measure) may have no special merit in terms of the end in view, and considerations of algebraic tractability may dictate our preference for the measure we adopt. There may then be no objection to the use of normalizing score transformations which would otherwise obscure the end in view.

In this context, we may recall that choice of the 
method of scoring involves considerations other than statistical. In so far as we interpret the advantages of substituting one treatment for another in terms of the health or survival of the individual, the taxonomic method will commend itself as the one more relevant to the humane intention of the trial; but it is not always convenient nor is it equally appropriate to costing the substitution vis $\grave{a}$ vis allocation of scarce resources. Growth is especially difficult to assess in terms referable to individual performance; and we shall commonly rely on a group mean or other representative score when growth is the criterion of efficacy, as in a dietetic trial. In some situations the claims of costing considerations may manifestly conflict with those of the individual, as when a treatment which lowers the mean duration of stay in hospital involves peculiar risks to a minority of patients.

The indisputably prior claim of taxonomic scoring in many types of trial has certain disadvantages from the view-point of the statistician. Any acceptable method of estimation in the taxonomic domain must prescribe the use of very large samples; and if it is true that we can formulate the distribution of a proportionate score difference referable to indefinitely large samples, we cannot as yet precisely specify how large they must be to justify our assurance that its use will not lead us astray.

We have seen that confidence intervals are specifiable with reference to a discrete variate, such as the proportionate score of an $r$-fold sample from the two-class universe (MODEL I (b)), only if we are content to express our uncertainty safeguard in the form $P_{f} \leqslant \alpha$. If the size of the sample is sufficiently large, we may invoke the normal approximation without sensible error. In that event, our uncertainty safeguard may permissibly take the form $P_{f}=\alpha$, and the solution of the problem will be as originally given by Wilson. When our concern is with a proportionate score difference, the assumption of normality does not suffice to assign an uncertainty safeguard $P_{f}=\alpha$ to statements about the limits between which the true value lies; but we shall now see that we can still work within the limitations of an uncertainty safeguard $P_{f} \leqslant \alpha$.

We shall here use the following symbols. We denote two treatment group efficacies by $p_{a}$ and $p_{b}$, their difference (the operational advantage of Treatment $B)$ being $M_{d}=\left(p_{b}-p_{a}\right)$. Our sample estimates of $p_{a}$ and $p_{b}$ will be respectively $p_{a . s}$ and $p_{b . s}$, the observed difference being $M_{d . s}=$ $\left(p_{b . s}-p_{a . s}\right)$. The true variance of the difference distribution referable to the $a$-fold sample (Treatment
Group $A$ ) and to the $b$-fold sample (Treatment Group B) is:

$$
\sigma_{d}^{2}=\frac{p_{a}\left(1-p_{a}\right)}{a}+\frac{p_{b}\left(1-p_{b}\right)}{b} .
$$

The normal approximation signifies that we may define as a normal variate of unit variance:

$$
\frac{M_{d . s}-M_{d}}{\sigma_{d}} \text {. }
$$

In so far as this is legitimate we may place $M_{d}$ as below in a confidence interval at $h \sigma$ level, i.e. a 5 per cent. uncertainty safeguard if $h=2$ :

$$
M_{d . s}-2 \sigma_{d}<M_{d}<M_{d . s}+2 \sigma_{d} \ldots .
$$

We can specify $\sigma_{d}$ precisely only if we know the numerical values of $p_{a}$ and $p_{b}$, in which event we should also know the exact value of $M_{d}$. Our problem arises because we have no such knowledge. Within the framework of the assumption that we are dealing with a normal variate, we must therefore modify the form of our statement, as we can do in two ways.

First, we may make use of the fact that $p(1-p)$ is a maximum if $p=\frac{1}{2}$, whence $\sigma_{d}^{2}$ cannot exceed the value it would have if $p_{a}=\frac{1}{2}=p_{b}$, in which event $M_{d}=0$. Accordingly, we shall define a statistic:

$$
\sigma_{d .5}^{2}=\frac{1}{4 a}+\frac{1}{4 b}=\frac{(a+b)}{4 a b} \ldots \ldots
$$

We may then frame a confidence rule in the form:

$$
M_{d . s}-2 \sigma_{d .5}<M_{d}<M_{d . s}+2 \sigma_{d .5} \ldots \text {. (ix) }
$$

Unless $M_{d}=0$, and then only if $p_{a}=\frac{1}{2}=p_{b}$, the interval of length $4 \sigma_{d .5}$ will be greater than the interval of length $4 \sigma_{d}$ and the appropriate uncertainty safeguard will accordingly be less, i.e. $P_{f} \leqslant 0.05$ for the specification of a confidence interval defined by (vii). If what we may usefully call the operational level of the trial is about 50 per cent, i.e. $\frac{1}{2}\left(p_{b}+p_{a}\right)=$ 0.5 , the uncertainty safeguard we can attach to a statement in terms of (ix) will not differ much from 0.05 . Otherwise, it may be much less. It is therefore instructive to examine the consequences of using it for the case of equal samples $a=r=b$. If $q_{a}=\left(1-p_{a}\right)$ and $q_{b}=\left(1-p_{b}\right)$, we then have:

$$
\sigma_{d}^{2}=\frac{p_{a} q_{a}+p_{b} q_{b}}{r} \text { and } \sigma_{d .5}{ }^{2}=\frac{1}{2 r} .
$$

For expository purposes, we shall now take a backstage view, i.e. we shall assume that we know the values of $p_{a}$ and $p_{b}$. To interpret our uncertainty safeguard precisely for a statement in the form prescribed by $(i x)$, we can then restate our confidence interval in terms of $\sigma_{d}$ by the substitution $h \sigma_{d}=$ $2 \sigma_{d .5}$, so that $h^{2}\left(p_{a} q_{a}+p_{b} q_{b}\right)=2$. If $M_{d}=0 \cdot 1$ 
(operational advantage 10 per cent.), we then have:

(i) $h=2.01$ and $P_{f}=0.044$, when $p_{a}=0.45$

(operational level 50 per cent.);

(ii) $h=2 \cdot 83$ and $P_{f}=0.0046$, when $p_{a}=0.1$

(operational level 15 per cent.).

Thus the upper limit we set to our uncertainty safeguard will be very conservative, in the sense that it may greatly understate the probability of erroneous assertion within the framework of the rule, when $p_{a}$ and $p_{b}$ lie near the limit of the range for which we may invoke the normal approximation with propriety for equal samples of less than 100 .

Since the rule prescribed by (ix) may thus lead us to be over-cautious, however large the sample we take, we may usefully explore an alternative procedure. We define an unbiassed estimate of $\sigma^{2}{ }_{d}$ based on sample values as:

$$
s_{d}^{2}=\frac{p_{a . s}\left(1-p_{a . s}\right)}{a-1}+\frac{p_{b . s}\left(1-p_{b . s}\right)}{b-1} .
$$

For large samples it will be sufficient to write:

$$
s_{d}=\frac{p_{a . s}\left(1-p_{a . s}\right)}{a}+\frac{p_{b . s}\left(1-p_{b . s}\right)}{b} \ldots
$$

We may then define an interval by:

$$
M_{d . s}-2 s_{d}<M_{d}<M_{d . s}+2 s_{d} \ldots \ldots(x i)
$$

If the size ( $a$ and $b$ ) of the samples we take is indefinitely large, $s_{d}$ will not differ sensibly from $\sigma_{d}$; and we can make an assertion in the form prescribed by (xi) with an uncertainty safeguard $P_{f}=\alpha=0.05$. Actually, we have to deal with finite samples, and cannot therefore state with assurance that $P_{f} \leqslant 0.05$ since the sample statistic $s_{d}$ may be less or greater than $\sigma_{d}$. All we can say with assurance is that we shall not often err greatly if our samples are large.

If we wish to design a trial to ensure that the confidence interval will be of length $C_{1}$, having no prior knowledge about the operational level as defined above, the best we can do is to use (ix), i.e.

$$
4 \sigma_{d . s}=C_{1}=\frac{2 \sqrt{a+b}}{\sqrt{\overline{a b}}} .
$$

It is advantageous to use treatment groups of equal size, because the proportionate score difference distribution approaches normality as we increase, $(a+b)$ more rapidly when $a=r=b$, in which event:

$$
r=\frac{8}{C_{1}^{2}}
$$

If the operational level of the trial is in the neighbourhood of 50 per cent., our uncertainty safeguard will then be less, but not greatly less, than 0.05 , for the assertion that $M_{d}$ lies in a 10 per cent. interval $\left(C_{1}=0 \cdot 1\right)$. All we can say in advance is that we should never need to use larger samples to justify the assertion $M_{d . s}-0.05<M_{d}<M_{d . s}+0.05$ with an uncertainty safeguard not greater than $0 \cdot 05$. However this procedure may prescribe samples of grossly excessive size if the operational level is near zero or near unity. Again, we may look at the issue heuristically. We suppose that $p_{a}=0 \cdot 1$ and $p_{b}=$ $0 \cdot 2$, so that $2 \sigma_{d}=r^{-2}$. If we set $C_{1}=4 \sigma_{d}$ we then have $r=4 C_{1}^{-2}$; and we shall require equal samples of 400 . If we have at our disposal information results of a pilot trial, we shall have all the information relevant for a point estimate of the operational level; and we may be content to compromise on a specification of $r$ referable to $(i x)$, and one referable to $(x i)$. We thus arrive at the following conclusions:

(i) if we wish to set our interval at a length sufficient to justify any useful assertion about the operational advantage of substituting Treatment $B$ for Treatment $A$, we shall commonly require samples sufficiently large to justify recourse to the normal approximation;

(ii) within the framework of normal quadrature as a computing device for summation of terms of a discrete distribution, and pending an approach to the problem by new methods which we hope to explore in a subsequent communication, we must confine ourselves either to assertions which may be over-cautious or to assertions which may be unduly favourable to the new treatment.

To get into focus the major issues raised by interval estimation in the domain of representative scoring we should bear in mind two considerations:

(a) the assumption that the normal distribution of unit variance tallies closely with that of the unit sample score expressed in standard form is highly gratuitous;

(b) even when such an assumption is grossly erroneous, the sample size need not be large to ensure a close normal fit for the distribution of the sample mean and that of the difference between the means of samples from different universes. The last statement is true even when the number of score classes of the u.s.d. is small. Thus the error in assigning the sum of terms in the range $\pm 2 \sigma_{m}$ by recourse to the normal integral with due regard to the half-interval correction is trivial for the 51 scoreclass distribution of the 10 -fold sample mean from a six-class rectangular universe (e.g. unbiassed cubical die).

It is necessary to emphasize the foregoing distinction for two reasons:

(i) we cannot make any general statement about how large an error we incur, if we invoke the $t$-distribution of the mean score of samples from a normal parent universe; 
(ii) the distribution of the difference between mean scores of samples from a normal universe is not itself a $t$-variate.

For any advantage we may derive in terms of economy of sample size by recourse to any method based on the $t$-distribution, we thus incur a liability to unspecifiable error arising from the necessary assumption that the relevant parent distribution is normal. For this reason, we propose to defer to a later contribution discussion concerning the merits of small sample methods. The ensuing remarks outline the theory of a procedure analogous to $(x i)$ above, like the latter admittedly inexact and subject to the same limitations.

We postulate two unit sample distributions $\left(A\right.$ and $B$ ) whose means are $M_{a}$ and $M_{b}$ and variances $\sigma_{a}{ }^{2}, \sigma_{b}{ }^{2}$. We then denote the sample means of sizeable $a$-fold samples from $A$, and $b$-fold samples from $B$ as $M_{a . s}$ and $M_{b . s}$, distributed respectively with variance $\sigma_{m}{ }^{2} \cdot a=a^{-1} \sigma_{a}^{2}$ and $\sigma_{m}^{2} . b=b^{-1} \sigma_{b}{ }^{2}$.

The variance of the distribution of the mean difference $\left(M_{d . s}\right)$ will then be $\sigma_{d}{ }^{2}=\sigma_{m}{ }^{2} \cdot a+\sigma_{m}{ }^{2} . b$. In this context we may assume that each constituent sample exceeds fifty. For reasons stated we may then without compunction postulate as a normal score of unit variance:

$$
\frac{M_{d . s}-M_{d}}{\sigma_{d}} .
$$

At $h \sigma$ confidence level $\left(P_{f}=\alpha\right)$, we may then define the interval:

$$
M_{d . s}-h \sigma_{d} \leqslant M_{d} \leqslant M_{d . s}+h \sigma_{d} \ldots \text { (xii) }
$$

In practice, we shall not know the value of $\sigma_{d}$. All we shall be able to specify is its unbiassed estimate based on unit sample scores $x_{a . n}, x_{b . n}, v i z$. :

$$
\begin{aligned}
s_{d}{ }^{2}=\frac{1}{a(a-1)} \sum_{n=1}^{n=a}\left(x_{a . n}-M_{a . s}\right)^{2}+ \\
\frac{1}{b(b-1)} \sum_{n=1}^{n=b}\left(x_{b . n}-M_{b . s}\right)^{2} \ldots(x i i i)
\end{aligned}
$$

If $a$ and $b$ are indefinitely large, we may thus define our confidence interval at $h \sigma$ level by:

$$
M_{d . s}-h s_{d} \leqslant M_{d} \leqslant M_{d . s}+h s_{d} \ldots \text { (xiv) }
$$

We cannot assign the uncertainty safeguard $P_{f}=\alpha$ to an assertion of this form if referable to finite samples; but we may anticipate that gross error will be rare, if we assign to it $P_{f} \simeq \alpha$ when $(a+b)>200$.

\section{Operational Advantage in Recorded Trials}

While the performance of a significance test which invokes a unique null hypothesis irrelevant to the humane intention of a trial is now a universal ritual in statistical assessment of therapeutic or of prophylactic trials, it is fair to record that some authors associate with an observed difference $\left(M_{d . s}\right)$ the statistics $s_{d}$ defined by expression $(x)$ above as an estimate of the range of the universe parameter $M_{d}$ deemed to be significant in accordance with the outcome of the test. As we have seen in Section 3 above, interval estimation subsumes a test procedure embracing any conceivably relevant hypothesis. Hence the performance of a significance test as a preliminary to specification of the estimated range of a difference is an entirely redundant undertaking which emphasizes how little the concept of interval estimation has penetrated the field under discussion.

In what follows, we shall use the published data of 24 trials to illustrate results of applying the statistics specified in Expressions (ix) and (xi). Table I cites the source and reference number of each trial. Table II (opposite) cites the objectives

\begin{tabular}{|c|c|c|c|}
\hline No. & Trial & Source & Date \\
\hline $\begin{array}{l}11 \\
12 \\
13 \\
14 \\
15 \\
\\
16 \\
\\
17 \\
18 \\
19 \\
20 \\
21 \\
22 \\
23 \\
24\end{array}$ & $\begin{array}{l}\text { B.T. Malaria } \\
\text { B.T. Malaria } \\
\text { Gonorrhoea } \\
\text { Gonorrhoea } \\
\text { Tuberculosis } \\
\text { (prophylactic) } \\
\text { Whooping cough } \\
\text { (prophylactic) } \\
\text { B.T. Malaria relapses } \\
\text { Tuberculosis } \\
\text { Tuberculosis } \\
\text { Influenza (prophylactic) } \\
\text { Gonorrhoea } \\
\text { Gonorrhoea } \\
\text { Gonorrhoea } \\
\text { Burns } \\
\text { Burns } \\
\text { Burns } \\
\text { Measles (prevention) } \\
\text { Measles (attentuation) } \\
\text { Tuberculosis } \\
\text { Tuberculosis } \\
\text { Tuberculosis } \\
\text { Enteric Fever 1907 (a) } \\
\text { Enteric Fever 1907 (b) } \\
\text { Common Cold }\end{array}$ & $\begin{array}{l}\text { War Office* } \\
\text { War Office } \\
\text { War Office } \\
\text { War Office } \\
\text { Aronson and Palmer } \\
\text { Medical Research Council } \\
\text { War Office } \\
\text { Medical Research Council } \\
\text { Medical Research Council } \\
\text { Francis, Salk, and } \\
\text { Quilligan } \\
\text { War Office } \\
\text { War Office } \\
\text { War Office } \\
\text { Jackson, Lowbury, and } \\
\text { Topley } \\
\text { Jackson, Lowbury, and } \\
\text { Topley } \\
\text { Jackson, Lowbury, and } \\
\text { Topley } \\
\text { Medical Research Council } \\
\text { Medical Research Council } \\
\text { Medical Research Council } \\
\text { Medical Research Council } \\
\text { Medical Research Council } \\
\text { Greenwood } \\
\text { Greenwood } \\
\text { Medical Research Council }\end{array}$ & $\begin{array}{l}1948 \\
1948 \\
1948 \\
1948 \\
1946 \\
1951 \\
1948 \\
1948 \\
1948 \\
1947 \\
1948 \\
1948 \\
1948 \\
1951 \\
1951 \\
1951 \\
1950 \mathrm{a} \\
1950 \mathrm{a} \\
1950 \mathrm{~b} \\
1950 \mathrm{~b} \\
1950 \mathrm{~b} \\
1935 \\
1935 \\
1944\end{array}$ \\
\hline
\end{tabular}
and yardsticks of efficacy. Table III (overleaf) presents the analysis.

TABLE I

SOURCE AND DATE OF 24 TRIALS

* "Statistical Report on the Health of the Army." H.M.S.O., London.

In Table III, the entry $100 p_{a . s}$ as the percentage efficacy of the yardstick treatment is intended to give some indication of the operational level of the trial on the basis of available evidence. This is pertinent to both the main issues raised in the foregoing discussion, viz.: 
TABLE II

TREATMENT AND CRITERION OF EFFICACY IN 24 TRIALS

\begin{tabular}{|c|c|c|c|c|}
\hline \multirow{2}{*}{ No. } & \multirow{2}{*}{ Trial } & \multicolumn{2}{|c|}{ Treatment } & \multirow{2}{*}{ Criterion of Efficacy } \\
\hline & & $\mathbf{A}$ & B & \\
\hline 1 & B.T. Malaria & Mepacrine & Quinine and pamaquin & No relapse \\
\hline 2 & B.T. Malaria & Mepacrine & Quinine and pamaquin & $\begin{array}{l}\text { Only one relapse among those } \\
\text { experiencing at least one }\end{array}$ \\
\hline 3 & Gonorrhoea & $\begin{array}{l}\text { Sulphathiazole or } \\
\text { sulphapyridine }\end{array}$ & Penicillin & No recourse to further treatment \\
\hline 4 & Gonorrhoea & $\begin{array}{l}\text { Sulphathiazole or } \\
\text { sulphapyridine }\end{array}$ & Penicillin & Permanent cure \\
\hline 5 & Tuberculosis (prophylactic) & Unvaccinated & BCG vaccination & Absence of disease \\
\hline 6 & Whooping cough (prophylactic) & Unvaccinated & Vaccinated & No attack \\
\hline 7 & B.T. Malaria relapses & Paludrine at different levels & Quinine and pamaquin & No relapse \\
\hline 8 & Tuberculosis & Bed-rest & Streptomycin & Clinical improvement (6 mths) \\
\hline 9 & Tuberculosis & Bed-rest & Streptomycin & Survival (11 mths) \\
\hline 10 & Influenza (prophylactic) & Unvaccinated & Vaccinated & No attack \\
\hline 11 & Gonorrhoea & Sulphathiazole only & Sulphathiazole and $\mathrm{KMNO}_{4}$ & No recourse to further treatment \\
\hline 12 & Gonorrhoea & Sulphathiazole only & Sulphathiazole and $\mathrm{HgOCN}$ & No recourse to further treatment \\
\hline 13 & Gonorrhoea & Sulphathiazole and $\mathrm{HgOCN}$ & Sulphathiazole and $\mathrm{KMnO}_{4}$ & No recourse to further treatment \\
\hline 14 & Burns & No polymixin & Polymixin & No infection with Ps. pyocyanea \\
\hline 16 & Burns & No polymixin & Polymixin & Good graft \\
\hline 17 & Measles (prevention) & Adult serum & Globulin (at two levels) & No attack \\
\hline 18 & Measles (attenuation) & Adult serum & Globulin (at two levels) & Modification of symptoms \\
\hline 19 & Tuberculosis & Bed-rest & Para-aminosalicylic acid & Clinical improvement (6 mths) \\
\hline 20 & Tuberculosis & Para-aminosalicylic acid & Streptomycin & $\begin{array}{l}\text { Marked radiological improvement } \\
\text { (6 mths) }\end{array}$ \\
\hline 21 & Tuberculosis & Streptomycin & $\begin{array}{l}\text { Streptomycin with Para- } \\
\text { aminosalicylic acid }\end{array}$ & Clinical improvement (6 mths) \\
\hline 22 & Enteric fever, $1907(a)$ & Uninoculated & Inoculated & No attack \\
\hline 23 & Enteric fever, 1907 (b) & Uninoculated & Inoculated & Survival \\
\hline 24 & Common cold & Control & Patulin & Cured or improved (48 hrs) \\
\hline
\end{tabular}

(i) the adequacy of the normal approximation;

(ii) in what circumstances the interval specified in accordance with the rule of procedure embodied in Expression (ix) is likely to lead to an excessively cautious specification of the interval length assignable in terms of the upper limit $(\alpha)$ of the uncertainty safeguard $\left(P_{f}\right)$.

For illustrative purposes we have hitherto defined the interval by $h=2$ in alternative statements:

Method I of (ix) above:

$$
M_{d . s}-h \sigma_{d .5} \leqslant M_{d} \leqslant M_{d . s}+h \sigma_{d .5},
$$

Method II of (xi) above:

$$
M_{d . s}-h s_{d} \leqslant M \leqslant M_{d . s}+h s_{d} .
$$

Table III exhibits the result of using each method for $h=1.64$ and $h=1.96$. In the limit, i.e. for indefinitely large samples, these respectively specify the uncertainty safeguards appropriate to Method II:

$P_{f}=0.05=\alpha$

for the one-sided assertion

$$
M_{d} \geqslant M_{d . s}-1 \cdot 64 s_{d},
$$

$P_{f}=0 \cdot 10=\alpha$

for the two-sided assertion

$$
M_{d . s}-1 \cdot 64 s_{d} \leqslant M_{d} \leqslant M_{d . s}+1 \cdot 64 s_{d} ;
$$

$P_{f}=0.025=\alpha$

for the one-sided assertion

$$
M_{d} \geqslant M_{d . s}-1 \cdot 96 s_{d} \text {, }
$$

$P_{f}=0.05=\alpha$

for the corresponding two-sided assertion.

In practice we have to deal with finite samples; and we can merely assert $P_{f} \simeq \alpha$. In different background situations, referable to different unknown values of $P_{a}$ and $P_{b}$, the application of the 
TABLE III

ANALYSIS OF RESULTS OF 24 TRIALS

\begin{tabular}{|c|c|c|c|c|c|c|c|c|c|}
\hline \multirow{2}{*}{ Trial } & \multicolumn{2}{|c|}{ Size of Treatment Group } & \multicolumn{3}{|c|}{ Interval based on $h=1.64$} & \multicolumn{3}{|c|}{ Interval based on $h=1.96$} & \multirow{2}{*}{$100 p_{a . s}$} \\
\hline & $\mathbf{A}$ & B & Method I & Method I & & Method I & Method I & & \\
\hline $\begin{array}{l}1 \\
2 \\
3 \\
4 \\
5 \\
6 \\
7 \\
8 \\
9 \\
10 \\
11 \\
12 \\
13 \\
14 \\
15 \\
16 \\
17 \\
18 \\
19 \\
20 \\
21 \\
22 \\
23 \\
24\end{array}$ & $\begin{array}{r}650 \\
221 \\
764 \\
764 \\
1,457 \\
3,757 \\
215 \\
52 \\
52 \\
7,615 \\
569 \\
569 \\
95 \\
76 \\
76 \\
55 \\
215 \\
123 \\
52 \\
59 \\
54 \\
220 \\
220 \\
680\end{array}$ & $\begin{array}{r}584 \\
60 \\
251 \\
251 \\
1,550 \\
3,801 \\
107 \\
55 \\
55 \\
10,328 \\
204 \\
95 \\
204 \\
64 \\
64 \\
39 \\
212 \\
67 \\
59 \\
54 \\
53 \\
430 \\
430 \\
668\end{array}$ & 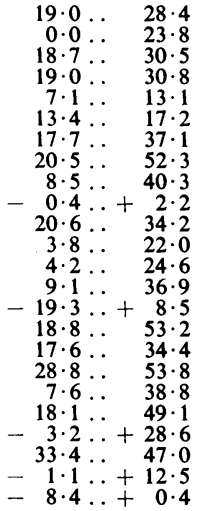 & $\begin{array}{r}20 \cdot 0 \ldots \\
5 \cdot 1 \ldots \\
20 \cdot 0 \ldots \\
19 \cdot 9 \ldots \\
8 \cdot 5 \ldots \\
14 \cdot 1 \ldots \\
19 \cdot 2 \ldots \\
21 \cdot 5 \ldots \\
9 \cdot 8 \ldots \\
0 \cdot 2 \ldots \\
22 \cdot 6 \ldots \\
5 \cdot 1 \ldots \\
6 \cdot 4 \ldots \\
11 \cdot 7 \ldots \\
18 \cdot 7 \ldots \\
20 \cdot 0 \ldots \\
18 \cdot 0 \ldots \\
32 \cdot 2 \ldots \\
8 \cdot 2 \ldots \\
19 \cdot 4 \ldots \\
0 \cdot 3 \ldots \\
34 \cdot 7 \ldots \\
3 \cdot 1 \ldots \\
7 \cdot 8 \ldots \\
\end{array}$ & $\begin{array}{l}27 \cdot 4 \\
18 \cdot 7 \\
29 \cdot 2 \\
29 \cdot 9 \\
11 \cdot 7 \\
16 \cdot 5 \\
35 \cdot 6 \\
51 \cdot 3 \\
39 \cdot 0 \\
1 \cdot 6 \\
32 \cdot 0 \\
20 \cdot 7 \\
22 \cdot 4 \\
34 \cdot 3 \\
7 \cdot 9 \\
52 \cdot 0 \\
33 \cdot 2 \\
50 \cdot 4 \\
38 \cdot 2 \\
47 \cdot 8 \\
25 \cdot 1 \\
45 \cdot 7 \\
8 \cdot 3 \\
0 \cdot 2\end{array}$ & 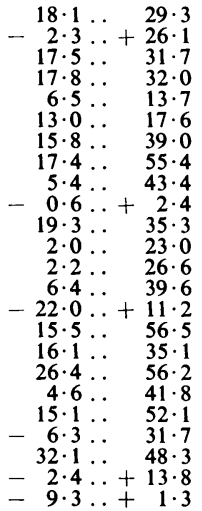 & $\begin{array}{r}19 \cdot 3 \ldots \\
3 \cdot 8 \ldots \\
19 \cdot 1 \ldots \\
18 \cdot 9 \ldots \\
8 \cdot 2 \ldots \\
13 \cdot 9 \ldots \\
17 \cdot 5 \ldots \\
18 \cdot 6 \ldots \\
7 \cdot 0 \ldots \\
0 \cdot 1 \ldots \\
21 \cdot 6 \ldots \\
3 \cdot 6 \ldots \\
4 \cdot 9 \ldots \\
9 \cdot 5 \ldots \\
21 \cdot 3 \ldots \\
16 \cdot 9 \ldots \\
16 \cdot 5 \ldots \\
30 \cdot 4 \ldots \\
5 \cdot 2 \ldots \\
16 \cdot 6 \ldots \\
2 \cdot 1 \ldots \\
33 \cdot 7 \ldots \\
2 \cdot 6 \ldots \\
8 \cdot 5 \ldots \\
-\end{array}$ & $\begin{array}{r}28 \cdot 1 \\
20 \cdot 0 \\
30 \cdot 1 \\
30 \cdot 9 \\
12 \cdot 0 \\
16 \cdot 7 \\
37 \cdot 3 \\
54 \cdot 2 \\
41 \cdot 8 \\
1 \cdot 7 \\
33 \cdot 0 \\
22 \cdot 2 \\
23 \cdot 9 \\
36 \cdot 5 \\
10 \cdot 5 \\
55 \cdot 1 \\
34 \cdot 7 \\
52 \cdot 2 \\
41 \cdot 2 \\
50 \cdot 6 \\
27 \cdot 5 \\
46 \cdot 7 \\
8 \cdot 8 \\
0 \cdot 5\end{array}$ & $\begin{array}{l}66 \cdot 0 \\
81 \cdot 4 \\
61 \cdot 9 \\
56 \cdot 0 \\
87 \cdot 3 \\
80 \cdot 8 \\
53 \cdot 0 \\
32 \cdot 7 \\
53 \cdot 8 \\
91 \cdot 9 \\
62 \cdot 9 \\
62 \cdot 9 \\
35 \cdot 8 \\
64 \cdot 5 \\
38 \cdot 2 \\
25 \cdot 5 \\
42 \cdot 8 \\
51 \cdot 2 \\
32 \cdot 7 \\
22 \cdot 0 \\
74 \cdot 1 \\
56 \cdot 8 \\
94 \cdot 1 \\
73 \cdot 0\end{array}$ \\
\hline
\end{tabular}

rule to finite samples will lead to different risks of false assertion, some greater and some less than $\alpha$; and this may lead us to an unduly optimistic assessment of the operational advantage. Our uncertainty safeguard takes the unequivocal form $P_{f} \leqslant \alpha$ if we use Method I, but the length of the two-sided confidence interval $2 h \sigma_{d .5}$ will be greater than the length of the confidence interval $2 h \sigma_{d}$ based on the true value of $\sigma_{d}$, and the value of $h$ which suffices to specify as $\alpha$ the upper limit of $P_{f}$. As explained above, the penalty of making our assertion overcautious in this sense will be less exacting if the true operational level of the test, elsewhere defined, is near 50 per cent. level. We cannot know the true operational level $50\left(p_{a}+p_{b}\right)$; but our sample statistic $100 p_{a . s}$ suffices to give what indication the figures can yield.

\section{SUMMARY}

(1) The operational intention of a prophylactic or a therapeutic trial is to assess the advantage of substituting one treatment (Treatment $B$ ) for another (Treatment $A$ ). This is commonly expressible in the form: by how much does the success rate or measure for Treatment $B$ exceed that for Treatment $A$ ?

(2) In the theory of inference we adopt, we aim at making statements which are true within the restriction of an uncertainty safeguard $\left(P_{f}\right)$ specifying an acceptable small probability of false assertion. Within these terms of reference two forms of statistical procedure are available. Of these, the method of interval estimation, alone can supply a terminal statement of a suitable form. The complete statement in the general case will then be that $P_{f} \leqslant \alpha$ for the assertion that the true difference $\left(M_{d}\right)$ lies between two values calculable from the data supplied by the trial.

(3) We may regard the procedure of interval estimation as the successive performance of a significance test to each of an infinitude of admissible hypotheses, including, of course, the conventional null hypothesis that the two treatments are of equal efficacy. Accordingly, the performance of a significance test referable to any such unique null hypothesis as a preliminary to estimation is redundant.

(4) Certain difficulties still beset the specification of a wholly satisfactory procedure of estimation appropriate to prohylactic and therapeutic trials. In the domain of taxonomic scoring, which is commonly more convenient and more consistent with the humane intention of the trial, an available method for assigning confidence intervals to a difference is accurate as the size of the samples becomes indefinitely large. In the representative domain, a method with the same limitations is also available. In each case further investigation is requisite to assess risks associated with the use of such approximate formulae.

(5) Objections to the use of approximate methods assume a less formidable aspect, when we give due 
consideration to the fact that the method of interval estimation can offer no prospect of locating within an interval of acceptable length associated with an acceptable uncertainty safeguard the operational advantage of substituting one treatment for another, unless we are indeed free to use large samples.

\section{ADDENDUM}

In replacing the appeal to the customary unique null hypothesis by the concept of operational advantage, we bring more clearly into focus an issue of outstanding importance with respect to the intention of any method of statistical assessment. When confronted with an interval estimate of the type here discussed, the administrator may well enquire in what circumstances he may regard it as relevant to policy. If the statistician replies that he has said all he can legitimately say about the relative merits of two treatment procedures on the basis of the evidence at his disposal, he may then refrain from asking how far the evidence at the disposal of the statistician is in fact relevant to unspecified circumstances incident to the trial. Though there is a deep cleavage between opposing schools of current thought, theoretical statisticians . with otherwise widely diverse views seem to make common cause in seeking interpretations consistent with two assumptions:

(a) with proper precautions we may regard each treatment group as a sample taken randomwise from an infinite population; (b) on the same understanding, the same homogeneous infinite population is the source of any other such sample chosen subsequently with the same precautions.

The circumstances in which such assumptions are more or less relevant to the use we make of an interval estimate are not self-evident nor immune from legitimate scrutiny; and we hope to examine in a later communication the propriety of invoking statistical theory as a basis for extrapolation beyond the limits of a clearly defined framework of repetition.

\section{REFERENCES}

Aronson, J. D., and Palmer, C. E. (1946). Publ. Hlth. Rep., Wash., 61,802 .

Clopper, C. J., and Pearson, E. S. (1934). Biometrika, 26, 404.

Fisher, R. A. (1936). Proc. Amer. Acad. Arts. Sci., 71, 245.

- (1949). "The Design of Experiments", 5th ed., pp. 13-14, 16. Oliver and Boyd, Edinburgh.

Francis, T., Salk, J. E., and Quilligan, J. J. (1947). Amer. J. publ. Hlth, 37, 1013 .

Greenwood, M. (1935). "Epidemics and Crowd Diseases." Williams

Hogben, L., and Wrighton, R. (1952). British Journal of Social Medicine, 6, 89 .

Jackson, D. M., Lowbury, E. J. C., and Topley, E. (1951). Lancet, $2,137$.

Medical Research Council (1944). Lancet, 2, 373.

- (1948). Brit. med. J., 2, 769.

(1950a). Lancet, 2,732

(1950b). Brit. med. J., 2, 1073.

(1950b). Brit. med. J.,

National Bureau of Standards (1949). "Tables of the Binomial Probability Distribution." Appl. Maths. Ser. No. 6. Govt. Printing Office, Washington.

Neyman, J. (1934). J. roy. statist. Soc., 97, 558.

Wald, W. (1947). "Sequential Analysis", p. 109. Wiley, New York

War Office (1948). "Statistical Report on the Health of the Army, 1943-45", introd. L. Hogben. H.M.S.O., London.

1943-45", introd. L. Hogben. H.M.S.O., London.
Wilson, E. B. (1927). J. Amer. statist. Ass., 22, 209.

Wilson, E. B. (1927). J. Amer. statist. Ass.
(1942). Proc. nat. Acad. Sci., 28, 88. 